\title{
Trichloro-oxazolines as activated donors for aminosugar coupling Supplementary material
}

\author{
Timothy. J. Donohoe, ${ }^{\S *}$ James G. Logan ${ }^{¥}$ and David Laffan ${ }^{\mathbb{I I}}$ \\ Dyson Perrins Laboratory, South Parks Road, Oxford, OX1 3QY, UK. \\ Department of Chemistry, University of Manchester, Oxford Road, Manchester, M13 \\ 9PL, UK. \\ AstraZeneca Pharmaceuticals, Silk Road Business Park, Macclesfield, SK10 2NA, \\ $U K$.
}

\section{General Procedure for ONAP Deprotection}

Triacetate 5 (or 13 or 18) $(0.580 \mathrm{~g}, 0.9817 \mathrm{mmol})$ was dissolved in DCM $(10 \mathrm{ml})$ and water (2\%, v/v) added. DDQ $(0.112 \mathrm{~g}, 0.4908 \mathrm{mmol})$ was added and the resulting reaction mixture stirred for 6 hours at room temperature. A further portion of DDQ $(0.112 \mathrm{~g}, 50 \mathrm{~mol} \%)$ was added to the reaction and stirring continued for a further 6 hours, after which DDQ $(0.25 \mathrm{~mol} \%)$ was added at 2 hourly intervals until complete consumption of starting material was indicated by TLC analysis. Analyses were performed at frequent intervals by removal of a small amount of the reaction mixture (typically $<1$ drop), partitioning of this between ethyl acetate $(0.5 \mathrm{ml})$ and saturated $\mathrm{NaHCO}_{3}(0.5 \mathrm{ml})$ allowed for TLC analysis of the organic component. The reaction was quenched with saturated $\mathrm{NaHCO}_{3}(50 \mathrm{ml})$ and extracted with ethyl acetate $(3 \times 100 \mathrm{ml})$. The organic extracts were combined and concentrated to $100 \mathrm{ml}$ under reduced pressure and washed successively with saturated $\mathrm{NaHCO}_{3}(3 \times 100 \mathrm{ml})$, water $(3 \times 100 \mathrm{ml})$, dried $\left(\mathrm{MgSO}_{4}\right)$ and concentrated under reduced pressure to afford the crude reaction products as a red oil. The crude lactol products were purified by column chromatography eluting with iso-hexane/ethyl acetate (4:1) to afford the lactols as a clear gum. These were normally used directly in the next step.

\section{Formation of trichloro-oxazolines}

The sugar lactol $(0.62 \mathrm{~g}, 1.38 \mathrm{mmol})$ dissolved in anhydrous acetonitrile $(25 \mathrm{ml})$, to this was added methane sulfonic anhydride $(0.36 \mathrm{~g}, 2.06 \mathrm{mmol})$ in one portion and the resulting reaction mixture was stirred for 20 minutes at room temperature under an atmosphere of nitrogen. Triethylamine $(0.42 \mathrm{~g}$, $4.1 \mathrm{mmol}$ ) was added and the reaction was stirred for a further hour, complete by TLC analysis. Saturated $\mathrm{NaHCO}_{3}(50 \mathrm{ml})$ was added and the crude products extracted with ethyl acetate $(3 \times 100 \mathrm{ml})$. The organic extracts were combined, dried $\left(\mathrm{Na}_{2} \mathrm{SO}_{4}\right)$ and concentrated under reduced pressure to afford 
a crude brown oil. The crude oil was purified by column chromatography eluting with iso-hexane/ethyl acetate $\left(5: 1, \mathrm{Et}_{3} \mathrm{~N} 1 \%\right)$ yielded the title trichloro-oxazoline as a clear resin.

Data for 6 :

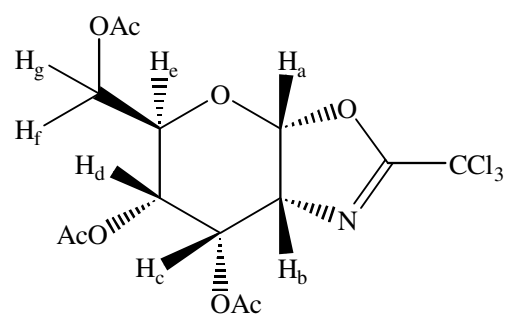

$v_{\max }($ film $) / \mathrm{cm}^{-1} 2955,1748,1657(\mathrm{C}=\mathrm{N}), 1370,1223,1061 ;[\alpha]_{\mathrm{D}}+36\left(\mathrm{c}=0.02, \mathrm{CHCl}_{3} @ 21^{\circ} \mathrm{C}\right)$; $\delta_{\mathrm{H}}\left(400 \mathrm{MHz} ; \mathrm{CDCl}_{3} ; \mathrm{Me}_{4} \mathrm{Si}\right) 6.38\left(1 \mathrm{H}, \mathrm{d},{ }^{3} J_{\mathrm{Ha}-\mathrm{Hb}} 7.6 \mathrm{~Hz}, \boldsymbol{H}_{\boldsymbol{a}}\right), 5.34-5.28\left(2 \mathrm{H}, \mathrm{m}, \boldsymbol{H}_{\boldsymbol{d}}, \boldsymbol{H}_{\boldsymbol{c}}\right), 4.56-4.52$ $\left(1 \mathrm{H}, \mathrm{m}, \boldsymbol{H}_{\boldsymbol{b}}\right), 4.28-4.25\left(2 \mathrm{H}, \mathrm{m}, \boldsymbol{H}_{\boldsymbol{f}}, \boldsymbol{H}_{\boldsymbol{g}}\right), 4.09\left(1 \mathrm{H}, \mathrm{ddd},{ }^{3} J_{\mathrm{He}-\mathrm{Hd}} 4.2 \mathrm{~Hz},{ }^{3} J_{\mathrm{He}-\mathrm{Hf}} 4.2 \mathrm{~Hz},{ }^{3} J_{\mathrm{He}-\mathrm{Hg}} 4.2 \mathrm{~Hz}\right.$, $\left.\boldsymbol{H}_{\boldsymbol{e}}\right), 2.12(3 \mathrm{H}, \mathrm{s}, \boldsymbol{M e}), 2.10(3 \mathrm{H}, \mathrm{s}, \boldsymbol{M e}), 2.08(3 \mathrm{H}, \mathrm{s}, \boldsymbol{M e}) ; \delta_{\mathrm{c}}\left(75 \mathrm{MHz} ; \mathrm{CDCl}_{3} ; \mathrm{Me}_{4} \mathrm{Si}\right)$ 170.4, 170.0, 169.9, 162.1, 104.4, 91.3, 71.6, 65.8, 65.0, 63.7, 63.0, 20.8, 20.7, 20.6; m/z (CI) 448 (50\%, $\left.\mathrm{M}+\left[\mathrm{NH}_{4}\right]^{+}\right), 431(100 \%, \mathrm{M}+1), 398(20 \%)$; HRMS calculated for $\mathrm{C}_{14} \mathrm{H}_{16} \mathrm{NO}_{8} \mathrm{Cl}_{3}$ requires 432.0020, found 432.0014 .

Data for 14:

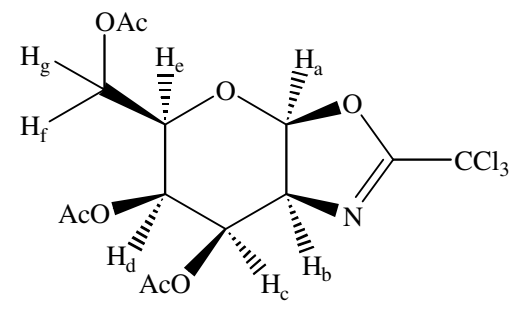

$v_{\max }($ film $) / \mathrm{cm}^{-1} 2944,1745,1664(\mathrm{C}=\mathrm{N}), 1228,1105,979 ;[\alpha]_{\mathrm{D}}-28\left(\mathrm{c}=1, \mathrm{CHCl}_{3} @ 25^{\circ} \mathrm{C}\right)$; $\delta_{\mathrm{H}}\left(500 \mathrm{MHz} ; \mathrm{CDCl}_{3} ; \mathrm{Me}_{4} \mathrm{Si}\right) 6.19\left(1 \mathrm{H}, \mathrm{d},{ }^{3} J_{\mathrm{Ha}-\mathrm{Hb}} 6.0 \mathrm{~Hz}, \boldsymbol{H}_{\boldsymbol{a}}\right), 5.45\left(1 \mathrm{H}, \mathrm{dd},{ }^{3} J_{\mathrm{Hd}-\mathrm{Hc}} 3.0 \mathrm{~Hz},{ }^{3} J_{\mathrm{Hd}-\mathrm{He}} 5.5\right.$ $\left.\mathrm{Hz}, \boldsymbol{H}_{\boldsymbol{d}}\right), 5.27\left(1 \mathrm{H}, \mathrm{dd},{ }^{3} J_{\mathrm{Hc}-\mathrm{Hd}} 3.0 \mathrm{~Hz},{ }^{3} J_{\mathrm{Hc}-\mathrm{Hb}} 6.0 \mathrm{~Hz}, \boldsymbol{H}_{\boldsymbol{c}}\right), 4.45\left(1 \mathrm{H}, \mathrm{dd},{ }^{3} J_{\mathrm{Hb}-\mathrm{Hc}} 6.0 \mathrm{~Hz},{ }^{3} J_{\mathrm{Hb}-\mathrm{Ha}} 6.0 \mathrm{~Hz}\right.$, $\left.\boldsymbol{H}_{\boldsymbol{b}}\right), 4.22-4.12\left(3 \mathrm{H}, \mathrm{m}, \boldsymbol{H}_{\boldsymbol{e}}, \boldsymbol{H}_{\boldsymbol{f}}, \boldsymbol{H}_{\boldsymbol{g}}\right), 2.15(3 \mathrm{H}, \mathrm{s}, \boldsymbol{M e}), 2.10(3 \mathrm{H}, \mathrm{s}, \boldsymbol{M e}), 2.05(3 \mathrm{H}, \mathrm{s}, \boldsymbol{M e}) ; \delta_{\mathrm{c}}(75 \mathrm{MHz} ;$ $\mathrm{CDCl}_{3} ; \mathrm{Me}_{4} \mathrm{Si}$ ) 170.30, 170.08, 162.00, 102.71, 71.29, 66.35, 65.24, 64.71, 62.24, 20.75, 20.59, 20.52; $m / z$ (EI) $432\left(40 \%, \mathrm{M}+[\mathrm{H}]^{+}\right), 398\left(90 \%, \mathrm{M}-[\mathrm{Cl}]^{-}\right)$; HRMS calculated for $\mathrm{C}_{14} \mathrm{H}_{16} \mathrm{NO}_{8} \mathrm{Cl}_{3}+[\mathrm{H}]^{+}$requires 432.0020, found 432.0023.

Data for 19: 


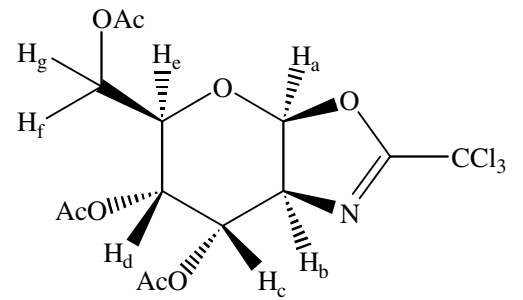

$v_{\max }\left(\right.$ film) $/ \mathrm{cm}^{-1} 2955,1748,1657(\mathrm{C}=\mathrm{N}), 1370,1223,1061 ;[\alpha]_{\mathrm{D}}+23\left(\mathrm{c}=0.01, \mathrm{CHCl}_{3} @ 23{ }^{\circ} \mathrm{C}\right) ; \delta_{\mathrm{H}}$ $\left(300 \mathrm{MHz} ; \mathrm{CDCl}_{3} ; \mathrm{Me}_{4} \mathrm{Si}\right) 6.19\left(1 \mathrm{H}, \mathrm{d},{ }^{3} J_{\mathrm{Ha}-\mathrm{Hb}} 5.7 \mathrm{~Hz}, \boldsymbol{H}_{\boldsymbol{a}}\right), 5.43\left(1 \mathrm{H}, \mathrm{dd},{ }^{3} J_{\mathrm{Hc}-\mathrm{Hb}} 5.7 \mathrm{~Hz},{ }^{3} J_{\mathrm{Hc}-\mathrm{Hd}} 5.7 \mathrm{~Hz}\right.$, $\left.\boldsymbol{H}_{\boldsymbol{c}}\right), 5.20\left(1 \mathrm{H}, \mathrm{dd},{ }^{3} J_{\mathrm{Hd}-\mathrm{Hc}} 5.7 \mathrm{~Hz},{ }^{3} J_{\mathrm{Hd}-\mathrm{He}} 8.1 \mathrm{~Hz}, \boldsymbol{H}_{\boldsymbol{d}}\right), 4.43\left(1 \mathrm{H}, \mathrm{t},{ }^{3} J_{\mathrm{Hb}-\mathrm{Ha}} 5.7 \mathrm{~Hz},{ }^{3} J_{\mathrm{Hb}-\mathrm{Hc}} 5.7 \mathrm{~Hz}, \boldsymbol{H}_{\boldsymbol{b}}\right)$, $4.28\left(2 \mathrm{H}, \mathrm{m}, \boldsymbol{H}_{\boldsymbol{f}}, \boldsymbol{H}_{\boldsymbol{g}}\right), 4.13\left(1 \mathrm{H}, \mathrm{m}, \boldsymbol{H}_{\boldsymbol{e}}\right), 2.15(3 \mathrm{H}, \mathrm{s}, \boldsymbol{M e}), 2.12(3 \mathrm{H}, \mathrm{s}, \boldsymbol{M e}), 2.00(3 \mathrm{H}, \mathrm{s}, \boldsymbol{M e})$; $\delta_{\mathrm{c}}\left(75 \mathrm{MHz} ; \mathrm{CDCl}_{3} ; \mathrm{Me}_{4} \mathrm{Si}\right) 170.51,169.51,169.28,162.84,102.40,71.99,68.09,67.69,66.86,63.41$, 20.75, 20.62 (2xMe); (EI) $432\left(50 \%, \mathrm{M}+[\mathrm{H}]^{+}\right)$; HRMS calculated for $\mathrm{C}_{14} \mathrm{H}_{16} \mathrm{NO}_{8} \mathrm{Cl}_{3}+[\mathrm{H}]^{+}$requires 432.0020, found 432.0025 .

\section{General Glycosylation Procedure}

Equimolar amounts of oxazoline glycosyl donor and glycosyl acceptors were dissolved in anhydrous DCM ( 5 Vol.) under an atmosphere of nitrogen. Pre-activated, powdered $4 \AA$ molecular sieves were added such that the reaction mixture became a slurry, but could still be stirred efficiently. The contents were cooled, and stirred for an hour before addition of -trifluoromethane-sulfonate (5 to $20 \mathrm{~mol} \%$ ). The reaction progress was monitored by TLC for consumption of starting materials. When complete consumption of starting materials was visualised the reaction was quenched with saturated $\mathrm{NaHCO}_{3}$. Triethylamine (2 to $10 \mathrm{ml}$ ) added to the reaction and the resultant mixture diluted with ethyl acetate $(100 \mathrm{ml})$. Molecular sieves were removed by filtration and washed with ethyl acetate $(100 \mathrm{ml})$. The organic components were then washed with saturated $\mathrm{NaHCO}_{3}(2 \times 100 \mathrm{ml})$, water $(3 \times 100 \mathrm{ml})$, dried $\left(\mathrm{MgSO}_{4}\right)$ and concentrated under reduced pressure to yield the crude products, which were purified by flash chromatography as appropriate.

\section{Compound 10}

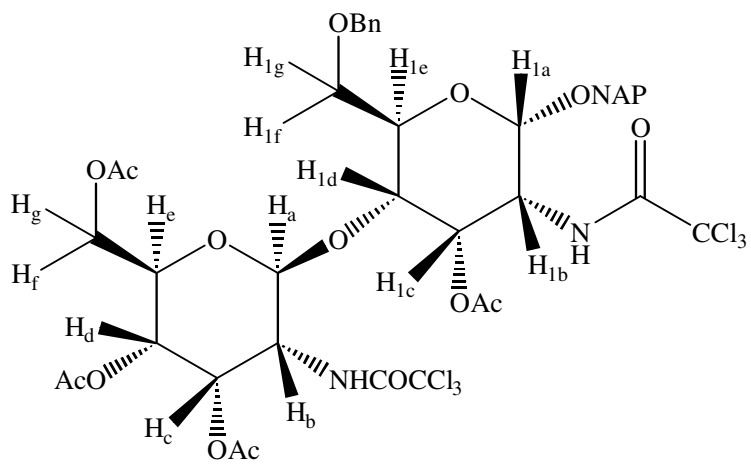


Trichloro-oxazoline glycosyl donor $6(1.0 \mathrm{~g}, 2.31 \mathrm{mmol})$ and C4-free alcohol glycosyl acceptor 7 $(1.38 \mathrm{~g}, 2.31 \mathrm{mmol})$ were dissolved in anhydrous DCM $(5 \mathrm{ml})$ and cooled to $0{ }^{\circ} \mathrm{C}$ under an atmosphere of argon. Pre-activated powdered molecular sieves $(4 \AA)$ were added such that the mixture became a slurry and the contents stirred at $0{ }^{\circ} \mathrm{C}$ for 1 hour. TMSOTf $(0.103 \mathrm{~g}, 0.463 \mathrm{mmol})$ was added and the reaction mixture stirred for 3 hours until complete by TLC analysis. Triethylamine ( $3 \mathrm{ml}$ ) was used to quench the reaction, which was subsequently diluted with ethyl acetate $(50 \mathrm{ml})$, the sieves removed by filtration and washed with ethyl acetate $(100 \mathrm{ml})$. The organic components were combined and washed with saturated $\mathrm{NaHCO}_{3}(3 \times 50 \mathrm{ml})$, water $(3 \times 75 \mathrm{ml})$, dried $\left(\mathrm{MgSO}_{4}\right)$ and concentrated under reduced pressure. The product was purified by column chromatography eluting with iso-hexane/ethyl acetate (1:1) yielding the title compound 10 as a clear gum $(1.98 \mathrm{~g}, 83 \%) . \quad v_{\max }(\mathrm{film}) / \mathrm{cm}^{-1} 34233344 \mathrm{br}$ $(\mathrm{NH}), \quad 2956,1752,1720,1508,1372,1229,1049,737 ;[\alpha]_{\mathrm{D}} 50 \quad\left(\mathrm{c}=1, \mathrm{CHCl}_{3} @ 23{ }^{\circ} \mathrm{C}\right)$; (Found C, 49.45; H, 4.16; N, 2.61\%, calculated for $\mathrm{C}_{42} \mathrm{H}_{44} \mathrm{~N}_{2} \mathrm{O}_{15} \mathrm{Cl}_{6}$ requires $\mathrm{C}, 49.00 ; \mathrm{H}, 4.31 ; \mathrm{N}$, $2.72 \%) ; \delta_{\mathrm{H}}\left(500 \mathrm{MHz} ; \mathrm{CDCl}_{3} ; \mathrm{Me}_{4} \mathrm{Si}\right)$ 7.88-7.74 (4H, m, NAPH), 7.55-7.31 (8H, m, Ph, NAPH), 7.23 $\left(\mathrm{d}, 1 \mathrm{H},{ }^{3} \boldsymbol{J}_{\mathrm{N} 1 \mathrm{H}-\mathrm{H} 1 \mathrm{~b}} 8.7 \mathrm{~Hz}, \mathrm{NH} \boldsymbol{H}_{1}\right), 6.85\left(1 \mathrm{H}, \mathrm{d},{ }^{3} \boldsymbol{J}_{\mathrm{NH}-\mathrm{Hb}} 8.1 \mathrm{~Hz}, \mathrm{NH}\right), 5.79\left(1 \mathrm{H}, \mathrm{dd},{ }^{3} \boldsymbol{J}_{\mathrm{H} 1 \mathrm{c}-\mathrm{H} 1 \mathrm{~d}} 3.2 \mathrm{~Hz}\right.$, $\left.{ }^{3} \boldsymbol{J}_{\mathrm{H} 1 \mathrm{c}-\mathrm{H} 1 \mathrm{~b}} 3.2 \mathrm{~Hz}, \boldsymbol{H}_{\boldsymbol{l c}}\right), 5.61\left(1 \mathrm{H}, \mathrm{dd},{ }^{3} J_{\mathrm{Hc}-\mathrm{Hb}} 3.0 \mathrm{~Hz},{ }^{3} J_{\mathrm{Hc}-\mathrm{Hd}} 3.0 \mathrm{~Hz}, \boldsymbol{H}_{\boldsymbol{c}}\right), 5.04\left(1 \mathrm{H}, \mathrm{d},{ }^{3} J_{\mathrm{H} 1 \mathrm{a}-\mathrm{H} 1 \mathrm{~b}}\right.$ $\left.4.5 \mathrm{~Hz}, \boldsymbol{H}_{\boldsymbol{l a}}\right), 4.97-4.88\left(3 \mathrm{H}, \mathrm{m}, \mathrm{OCH}_{2} \mathrm{NAP}, \boldsymbol{H}_{\boldsymbol{a}}, \boldsymbol{H}_{\boldsymbol{d}}\right), 4.74-4.68\left(2 \mathrm{H}, \mathrm{m}, \mathrm{OCH}_{2} \mathrm{NAP}, \mathrm{OCH} \boldsymbol{H}_{2} \mathrm{Ph}\right), 4.56$ $\left(1 \mathrm{H}, \mathrm{d},{ }^{2} J_{\mathrm{H}-\mathrm{H}} 11.8 \mathrm{~Hz}, \mathrm{OCH}_{2} \mathrm{Ph}\right), 4.31-4.01\left(7 \mathrm{H}, \mathrm{m}, \boldsymbol{H}_{\boldsymbol{l} \boldsymbol{b}}, \boldsymbol{H}_{\boldsymbol{l d}}, \boldsymbol{H}_{\boldsymbol{l}}, \boldsymbol{H}_{\boldsymbol{b}}, \boldsymbol{H}_{\boldsymbol{e}}, \boldsymbol{H}_{\boldsymbol{f}}, \boldsymbol{H}_{\boldsymbol{g}}\right), 3.88\left(1 \mathrm{H}, \mathrm{dd},{ }^{3} J_{\mathrm{Hlf}-}\right.$ H1e $\left.3.6 \mathrm{~Hz},{ }^{3} J_{\mathrm{H} 1 \mathrm{f}-\mathrm{H} 1 \mathrm{~g}} 11.8 \mathrm{~Hz}, \boldsymbol{H}_{1 f}\right), 3.63\left(1 \mathrm{H}, \mathrm{dd},{ }^{3} J_{\mathrm{H} 1 \mathrm{~g}-\mathrm{H} 1 \mathrm{e}} 3.6 \mathrm{~Hz},{ }^{3} J_{\mathrm{H} 1 \mathrm{~g}-\mathrm{H} 1 \mathrm{f}} 11.8 \mathrm{~Hz}, \boldsymbol{H}_{1 g}\right), 2.14(3 \mathrm{H}, \mathrm{s}$, Me), $2.13(3 \mathrm{H}, \mathrm{s}, \boldsymbol{M e}), 2.02(3 \mathrm{H}, \mathrm{s}, \boldsymbol{M e}), 1.98(3 \mathrm{H}, \mathrm{s}, \boldsymbol{M e}) ; \delta_{\mathrm{c}}\left(75 \mathrm{MHz} ; \mathrm{CDCl}_{3} ; \mathrm{Me}_{4} \mathrm{Si}\right)$ 170.9, 170.1, $169.1,169.0,161.2,161.1,137.8,134.3,133.1,133.0,128.4,128.3,127.9,127.7,127.7,127.5,126.4$, 126.2, 125.2, 99.9, 95.0, 92.1, 92.0, 74.1, 74.0, 70.4, 69.9, 68.9, 68.7, 68.0, 66.4, 62.3, 52.8, 50.5, 20.8, 20.7, 20.4, 20.3; m/z $\left(\mathrm{ES}^{+}\right) 1065\left(100 \%, \mathrm{M}+[\mathrm{K}]^{+}\right), 1027(80 \%, \mathrm{M}+1), 900(25 \%), 561(30 \%), 448$ $(40 \%)$; 


\section{Compound 11}

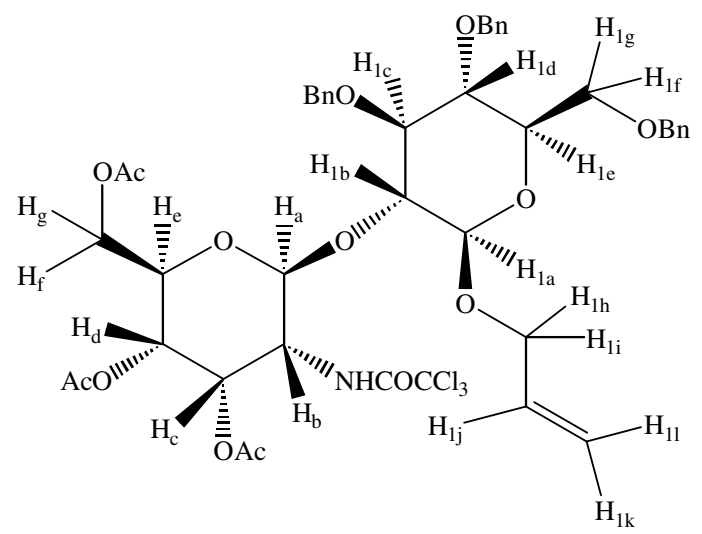

Allosamine donor $6(0.070 \mathrm{~g}, 0.1618 \mathrm{mmol})$ and C2-OH glucose glycosyl acceptor $11(0.095 \mathrm{~g}$, $0.1942 \mathrm{mmol}$ ) were coupled using the glycosidation procedure The product was purified by column chromatography eluting with iso-hexane/ethyl acetate (6:1) yielding the title compound $\mathbf{1 1}$ as a waxy $\operatorname{resin}(0.127 \mathrm{~g}, 85 \%) .[\alpha]_{\mathrm{D}}-13\left(\mathrm{c}=0.03, \mathrm{CHCl}_{3} @ 23^{\circ} \mathrm{C}\right) ; v_{\max }($ film $) / \mathrm{cm}^{-1} 3424,3030,2929,2872$, 1753, 1724, 1513, 1369, 1224, 1049, 840, 738; $\delta_{\mathrm{H}}\left(400 \mathrm{MHz} ; \mathrm{CDCl}_{3} ; \mathrm{Me}_{4} \mathrm{Si}\right)$ 7.30-7.17 (13H, m, Ph), $7.02(2 \mathrm{H}, \mathrm{m}, \boldsymbol{P h}), 6.70\left(1 \mathrm{H}, \mathrm{d},{ }^{3} J_{\mathrm{NH}-\mathrm{Hb}} 8.5 \mathrm{~Hz}, \boldsymbol{N H}\right), 5.90\left(1 \mathrm{H}, \mathrm{m}, \boldsymbol{H}_{\boldsymbol{k}}\right), 5.54\left(1 \mathrm{H}, \mathrm{t},{ }^{3} J_{\mathrm{Hc}-\mathrm{Hd}} 3.20 \mathrm{~Hz}\right.$, $\left.{ }^{3} J_{\mathrm{Hc}-\mathrm{Hb}} 3.20 \mathrm{~Hz}, \boldsymbol{H}_{\boldsymbol{c}}\right), 5.29\left(1 \mathrm{H}, \mathrm{dd},{ }^{3} J_{\mathrm{H} 11-\mathrm{H} 1 \mathrm{k}} 1.7 \mathrm{~Hz},{ }^{3} J_{\mathrm{H} 11-\mathrm{H} 1 \mathrm{j}} 17.0 \mathrm{~Hz}, \boldsymbol{H}_{1 l}\right), 5.35\left(1 \mathrm{H}, \mathrm{d}, 7.22 \mathrm{~Hz}, \boldsymbol{H}_{\boldsymbol{a}}\right)$, $5.22\left(1 \mathrm{H}, \mathrm{dd},{ }^{3} J_{\mathrm{H} 1 \mathrm{k}-\mathrm{H} 11} 1.7 \mathrm{~Hz},{ }^{3} J_{\mathrm{H} 1 \mathrm{k}-\mathrm{H} 1 \mathrm{j}} 10.5 \mathrm{~Hz}, \boldsymbol{H}_{\boldsymbol{l}}\right), 5.00\left(1 \mathrm{H}, \mathrm{dd},{ }^{3} J_{\mathrm{Hd}-\mathrm{Hc}} 3.1 \mathrm{~Hz},{ }^{3} J_{\mathrm{Hd}-\mathrm{He}} 8.5 \mathrm{~Hz}, \boldsymbol{H}_{\boldsymbol{d}}\right)$, $4.84\left(1 \mathrm{H}, \mathrm{d},{ }^{2} J_{\mathrm{H}-\mathrm{H}} 11.9 \mathrm{~Hz}, \mathrm{OCH}_{2} \mathrm{Ph}\right), 4.75\left(1 \mathrm{H}, \mathrm{d},{ }^{2} J_{\mathrm{H}-\mathrm{H}} 11.9 \mathrm{~Hz}, \mathrm{OCH} \boldsymbol{H}_{2} \mathrm{Ph}\right), 4.60-4.42(4 \mathrm{H}, \mathrm{m}$, $\left.4 \mathrm{xOCH} \boldsymbol{H}_{2} \mathrm{Ph}\right), 4.41\left(1 \mathrm{H}, \mathrm{d},{ }^{3} J_{\mathrm{H} 1 \mathrm{a}-\mathrm{H} 1 \mathrm{~b}} 7.5 \mathrm{~Hz}, \boldsymbol{H}_{1 \boldsymbol{a}}\right), 4.37\left(1 \mathrm{H}, \mathrm{m}, \boldsymbol{H}_{\boldsymbol{i}}\right), 4.24-4.05\left(4 \mathrm{H}, \mathrm{m}, \boldsymbol{H}_{\boldsymbol{b}}, \boldsymbol{H}_{f}, \boldsymbol{H}_{\boldsymbol{g}}, \boldsymbol{H}_{j}\right)$, $3.96\left(1 \mathrm{H}, \mathrm{m}, \boldsymbol{H}_{\boldsymbol{e}}\right), 3.70-3.53\left(5 \mathrm{H}, \mathrm{m}, \boldsymbol{H}_{l \boldsymbol{b}}, \boldsymbol{H}_{l \boldsymbol{c}}, \boldsymbol{H}_{l d}, \boldsymbol{H}_{l f}, \boldsymbol{H}_{l g}\right), 3.40\left(1 \mathrm{H}, \mathrm{m}, \boldsymbol{H}_{l e}\right), 2.02(3 \mathrm{H}, \mathrm{s}, \boldsymbol{M e})$, $1.95(3 \mathrm{H}, \mathrm{s}, \boldsymbol{M e}), 1.90(3 \mathrm{H}, \mathrm{s}, \boldsymbol{M e}) ; \delta_{\mathrm{c}}\left(75 \mathrm{MHz} ; \mathrm{CDCl}_{3} ; \mathrm{Me}_{4} \mathrm{Si}\right)$ 170.61, 169.14, 169.03, 161.25, $138.32,138.00,137.72,133.98,128.38,128.33,127.83,127.77,127.73,127.62,127.42,126.78$, 116.78, 100.72, 98.94, 92.22, 84.32, 79.23, 77.99, 76.68, 74.77, 74.71, 74.55, 73.46, 71.21, 69.9, 68.59, 67.16, 66.68, 62.67, 52.76, 20.74, 20.56, 20.32; m/z (APCI) $922\left(80 \%, \mathrm{M}+[\mathrm{H}]^{+}\right), 958(50 \%$, $\mathrm{M}+\left[\mathrm{Cl}^{-}\right)$.

\section{Compound 12}

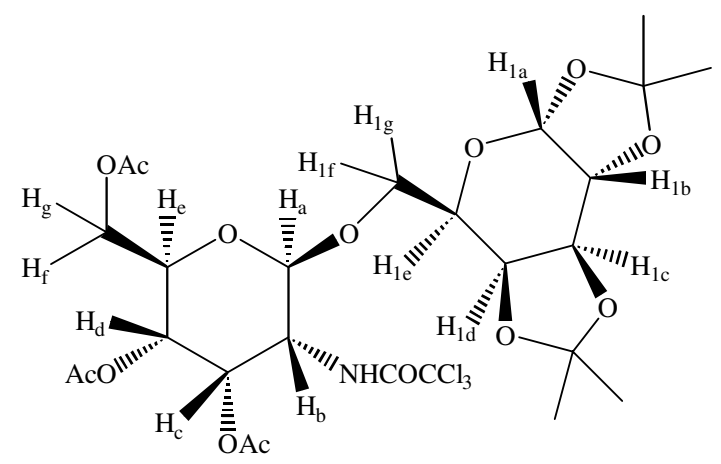


Trichloro-oxazoline glycosyl donor $6(0.225 \mathrm{~g}, 0.520 \mathrm{mmol})$ and C6-OH galactopyranose glycosyl acceptor $12(0.162 \mathrm{~g}, 0.624 \mathrm{mmol})$ were coupled using the glycosidation procedure already described The product was purified by column chromatography eluting with iso-hexane/ethyl acetate (4:1) yielding the title compound 12 as a white solid $(0.313 \mathrm{~g}, 87 \%)$. Mp $134{ }^{\circ} \mathrm{C} ; v_{\max }($ film $) / \mathrm{cm}^{-1} 3339$, $2988,2937,1751,1724,1530,1373,1233,1173,1069,1042,1008,917,901,821 ;[\alpha]_{\mathrm{D}}-44$ (c=0.02, $\mathrm{CHCl}_{3} @ 22{ }^{\circ} \mathrm{C}$ ); (Found C, 45.08; H, 5.31; N, 1.99\%, calculated for $\mathrm{C}_{26} \mathrm{H}_{36} \mathrm{NO}_{14} \mathrm{Cl}_{3}$ requires C, 45.07; H, 5.24; N, 2.02\%); $\delta_{\mathrm{H}}\left(400 \mathrm{MHz} ; \mathrm{CDCl}_{3} ; \mathrm{Me}_{4} \mathrm{Si}\right) 6.91\left(1 \mathrm{H}, \mathrm{d},{ }^{3} J_{\mathrm{NH}-\mathrm{Hb}} 7.6 \mathrm{~Hz}, \boldsymbol{N H}\right), 5.75$ $\left(1 \mathrm{H}, \mathrm{t},{ }^{3} J_{\mathrm{Hc}-\mathrm{Hb}} 3.2 \mathrm{~Hz},{ }^{3} J_{\mathrm{Hc}-\mathrm{Hd}} 3.2 \mathrm{~Hz} \boldsymbol{H}_{\boldsymbol{c}}\right), 5.50\left(1 \mathrm{H}, \mathrm{d},{ }^{3} \boldsymbol{J}_{\mathrm{H} 1 \mathrm{a}-\mathrm{H} 1 \mathrm{~b}} 5.2 \mathrm{~Hz}, \boldsymbol{H}_{\boldsymbol{l a}}\right), 5.20\left(1 \mathrm{H}, \mathrm{dd},{ }^{3} J_{\mathrm{Hd}-\mathrm{Hc}} 3.2\right.$ $\left.\mathrm{Hz},{ }^{3} J_{\mathrm{Hd}-\mathrm{He}} 8.8 \mathrm{~Hz}, \boldsymbol{H}_{\boldsymbol{d}}\right), 4.92\left(1 \mathrm{H}, \mathrm{d},{ }^{3} J_{\mathrm{Ha}-\mathrm{Hb}} 7.6 \mathrm{~Hz}, \boldsymbol{H}_{\boldsymbol{a}}\right), 4.61\left(1 \mathrm{H}, \mathrm{d},{ }^{3} J_{\mathrm{H} 1 \mathrm{c}-\mathrm{Hid}} 2.4 \mathrm{~Hz},{ }^{3} J_{\mathrm{H} 1 \mathrm{c}-\mathrm{Hib}} 8.4 \mathrm{~Hz}\right.$, $\left.\boldsymbol{H}_{\boldsymbol{l c}}\right), 4.30\left(3 \mathrm{H}, \mathrm{m}, \boldsymbol{H}_{\boldsymbol{f}}, \boldsymbol{H}_{\boldsymbol{g}}, \boldsymbol{H}_{\boldsymbol{l b}},\right), 4.20,\left(1 \mathrm{H}, \mathrm{m}, \boldsymbol{H}_{\boldsymbol{l d}}\right), 4.15\left(1 \mathrm{H}, \mathrm{m}, \boldsymbol{H}_{\boldsymbol{b}}\right), 4.09\left(2 \mathrm{H}, \mathrm{m}, \boldsymbol{H}_{\boldsymbol{e}}, \boldsymbol{H}_{f}\right), 3.96(1 \mathrm{H}$, $\left.\mathrm{m}, \boldsymbol{H}_{\boldsymbol{l}}\right), 3.68\left(1 \mathrm{H}, \mathrm{dd},{ }^{3} \boldsymbol{J}_{\mathrm{Hg}-\mathrm{He}} 1.5 \mathrm{~Hz},{ }^{2} J_{\mathrm{Hf}-\mathrm{Hg}} 12.5 \mathrm{~Hz}, \boldsymbol{H}_{g}\right), 2.10(3 \mathrm{H}, \mathrm{s}, \boldsymbol{M e}), 2.08(3 \mathrm{H}, \mathrm{s}, \boldsymbol{M e}), 2.01(3 \mathrm{H}$, s, Me), $1.49(3 \mathrm{H}, \mathrm{s}, \boldsymbol{M e}), 1.45(3 \mathrm{H}, \mathrm{s}, \boldsymbol{M e}), 1.35(3 \mathrm{H}, \mathrm{s}, \boldsymbol{M e}), 1.30(3 \mathrm{H}, \mathrm{s}, \boldsymbol{M e}) ; \delta_{\mathrm{c}}\left(75 \mathrm{MHz} \mathrm{CDCl}_{3}\right.$; $\left.\mathrm{Me}_{4} \mathrm{Si}\right)$ 170.57, 169.14, 168.97, 161.47, 109.25, 108.63, 99.73, 96.08, 71.26, 70.95, 70.49, 70.31, 69.05, 67.30, 66.75, 66.54, 62.37, 52.60, 26.05, 25.85, 24.87, 24.13, 20.69, 20.54, 20.49; $\mathrm{m} / \mathrm{z}$ (CI) 709 $\left(10 \%, \mathrm{M}+\left[\mathrm{NH}_{4}\right]^{+}\right)$; HRMS calculated for $\mathrm{C}_{26} \mathrm{H}_{36} \mathrm{NO}_{14} \mathrm{Cl}_{3}+\left[\mathrm{NH}_{4}\right]^{+}$requires 709.1545, found 709.1535.

\section{Compound 15}

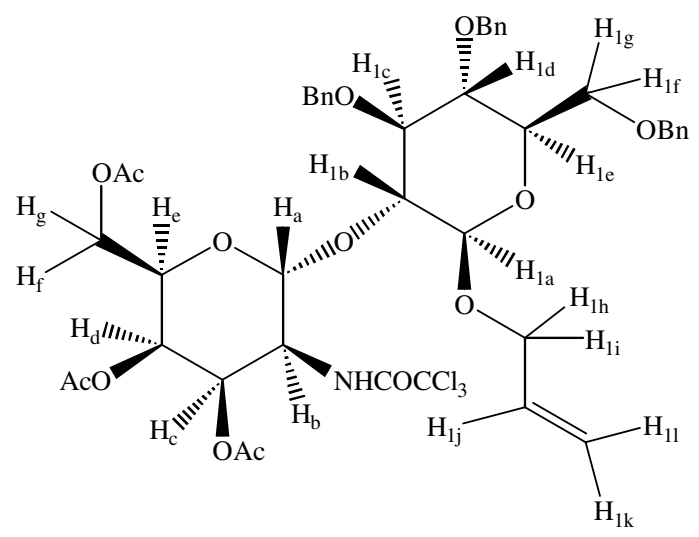

Talosamine trichloro-oxazoline glycosyl donor $14(0.115 \mathrm{~g}, 0.266 \mathrm{mmol})$ and C2-glucose glycosyl acceptor $\mathbf{8}$ (0.156 g, $0.3190 \mathrm{mmol})$ were coupled using the glycosidation procedure already described The product was purified by column chromatography eluting with iso-hexane/ethyl acetate (6:1) yielding the title compound $\mathbf{1 5}$ as a waxy resin $(0.21 \mathrm{~g}, 85 \%) .[\alpha]_{\mathrm{D}} 31\left(\mathrm{c}=0.01, \mathrm{CHCl}_{3} @ 22{ }^{\circ} \mathrm{C}\right) ; v_{\max }$ (film) $/ \mathrm{cm}^{-1} 3418,3063,3030,2925,1754,1722,1515,1454,1370,1228,1063$, 836, 820; (Found C, $57.39 ; \mathrm{H}, 5.21 ; \mathrm{N}, 1.62 \%$, calculated for $\mathrm{C}_{44} \mathrm{H}_{50} \mathrm{NO}_{14} \mathrm{Cl}_{3}$ requires $\mathrm{C}, 57.24 ; \mathrm{H}, 5.46 ; \mathrm{N}, 1.52 \%$ ); $\delta_{\mathrm{H}}\left(500 \mathrm{MHz} ; \mathrm{CDCl}_{3} ; \mathrm{Me}_{4} \mathrm{Si}\right) 7.40\left(1 \mathrm{H}, \mathrm{d},{ }^{3} \boldsymbol{J}_{\mathrm{NH}-\mathrm{Hb}} 9.9 \mathrm{~Hz}, \boldsymbol{N H}\right), 7.30-7.17(13 \mathrm{H}, \mathrm{m}, \boldsymbol{P h}), 7.02$ (2H, m, $\boldsymbol{P h}), 5.85\left(1 \mathrm{H}, \mathrm{m}, \boldsymbol{H}_{1 j}\right), 5.45\left(1 \mathrm{H}, \mathrm{s}, \boldsymbol{H}_{\boldsymbol{a}}\right), 5.21-5.10\left(3 \mathrm{H}, \mathrm{m}, \boldsymbol{H}_{\boldsymbol{d}}, \boldsymbol{H}_{\boldsymbol{l}}, \boldsymbol{H}_{1 l}\right), 4.96\left(1 \mathrm{H}, \mathrm{d},{ }^{2} J_{\mathrm{H}-\mathrm{H}} 11.25 \mathrm{~Hz}\right.$, 
$\left.\mathrm{OCH}_{2} \mathrm{Ph}\right), 4.88\left(1 \mathrm{H}, \mathrm{s}, \boldsymbol{H}_{\boldsymbol{c}}\right), 4.74\left(1 \mathrm{H}, \mathrm{d},{ }^{2} J_{\mathrm{H}-\mathrm{H}} 10.7 \mathrm{~Hz}, \mathrm{OC} \boldsymbol{H}_{2} \mathrm{Ph} \mathrm{Hz}\right), 4.62\left(1 \mathrm{H}, \mathrm{d},{ }^{2} J_{\mathrm{H}-\mathrm{H}} 11.25 \mathrm{~Hz}\right.$, $\left.\mathrm{OCH}_{2} \mathrm{Ph}\right), 4.57\left(1 \mathrm{H}, \mathrm{d},{ }^{2} J_{\mathrm{H}-\mathrm{H}} 12.3 \mathrm{~Hz}, \mathrm{OC} \boldsymbol{H}_{2} \mathrm{Ph}\right), 4.54\left(1 \mathrm{H}, \mathrm{d},{ }^{2} J_{\mathrm{H}-\mathrm{H}} 11.25 \mathrm{~Hz}, \mathrm{OC} \boldsymbol{H}_{2} \mathrm{Ph}\right), 4.48(1 \mathrm{H}, \mathrm{d}$, $\left.{ }^{2} J_{\mathrm{H}-\mathrm{H}} 11.3 \mathrm{~Hz}, \mathrm{OC} \boldsymbol{H}_{2} \mathrm{Ph}\right), 4.37\left(1 \mathrm{H}, \mathrm{d},{ }^{3} \boldsymbol{J}_{\mathrm{H} 1 \mathrm{a}-\mathrm{H} 1 \mathrm{~b}} 7.15 \mathrm{~Hz}, \boldsymbol{H}_{\boldsymbol{l a}}\right), 4.30\left(3 \mathrm{H}, \mathrm{m}, \boldsymbol{H}_{\boldsymbol{b}}, \boldsymbol{H}_{\boldsymbol{g}}, \boldsymbol{H}_{\boldsymbol{l} \boldsymbol{h}}\right), 4.05(1 \mathrm{H}, \mathrm{dd}$, $\left.{ }^{2} J_{\mathrm{H}-\mathrm{H}} 12.7 \mathrm{~Hz},{ }^{3} J_{\mathrm{Hi}-\mathrm{Hj}} 6.5 \mathrm{~Hz}, \boldsymbol{H}_{\boldsymbol{I i}}\right), 3.82\left(1 \mathrm{H}, \mathrm{dd},{ }^{2} J_{\mathrm{H}-\mathrm{H}} 11.1 \mathrm{~Hz},{ }^{3} J_{\mathrm{Hf}-\mathrm{He}} 6.6 \mathrm{~Hz}, \boldsymbol{H}_{\boldsymbol{e}}\right), 3.70-3.50(6 \mathrm{H}, \mathrm{m}$, $\left.\boldsymbol{H}_{\boldsymbol{e}}, \boldsymbol{H}_{\boldsymbol{l} \boldsymbol{b}}, \boldsymbol{H}_{\boldsymbol{l c}}, \boldsymbol{H}_{\boldsymbol{l d}}, \boldsymbol{H}_{\boldsymbol{l f}}, \boldsymbol{H}_{\boldsymbol{l g}}\right), 3.38\left(1 \mathrm{H}, \mathrm{d},{ }^{3} J_{\mathrm{H} 1 \mathrm{e}-\mathrm{H} 1 \mathrm{~d}} 9.3 \mathrm{~Hz}, \boldsymbol{H}_{\boldsymbol{l e}}\right), 2.06(3 \mathrm{H}, \mathrm{s}, \boldsymbol{M e}), 1.95(3 \mathrm{H}, \mathrm{s}, \boldsymbol{M e}), 1.92$ $(3 \mathrm{H}, \mathrm{s}, \mathbf{M e}) ; \delta_{\mathrm{c}}\left(100 \mathrm{MHz} ; \mathrm{CDCl}_{3} ; \mathrm{Me}_{4} \mathrm{Si}\right) 170.63,169.70,169.5,162.11,138.38,138.06,133.88$, $128.95,128.90,128.81,128.37,128.26,128.13,127.93,118.66,102.59,98.16,83.66,79.09,77.68$, 75.95, 75.36, 73.96, 70.90, 68.82, 67.69, 66.58, 65.05, 61.73, 50.53, 21.18, 21.08, 20.94; $m / z\left(\mathrm{ES}^{+}\right)$ $945\left(95 \%, \mathrm{M}+[\mathrm{Na}]^{+}\right)$.

\section{Compound 16}

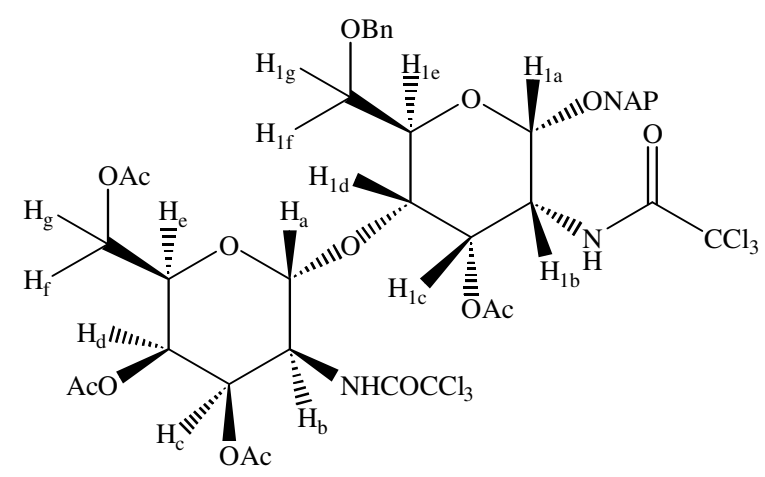

Trichloro-oxazoline glycosyl donor $14(0.092 \mathrm{~g}, 0.21 \mathrm{mmol})$ and C4-OH glycosyl acceptor 7 (0.127 g, $0.21 \mathrm{mmol}$ ) were coupled using the glycosidation procedure already. The product was purified by column chromatography eluting with iso-hexane/ethyl acetate (4:1) yielding the title compound $\mathbf{1 6}$ as a white solid (0.188 g, 86\%). Mp $119^{\circ} \mathrm{C} ; \mathrm{v}_{\max }\left(\right.$ film)/ $\mathrm{cm}^{-1} 3424,3349,2934,1742,1720,1508,1370$, 1230, 1060, 819; $[\alpha]_{\mathrm{D}}+62\left(\mathrm{c}=0.005, \mathrm{CHCl}_{3} @ 22{ }^{\circ} \mathrm{C}\right) ;($ Found C, 49.12; H, 4.17; N, 2.65\%, calculated for $\mathrm{C}_{42} \mathrm{H}_{44} \mathrm{~N}_{2} \mathrm{O}_{15} \mathrm{Cl}_{6}$ requires $\left.\mathrm{C}, 49.00 ; \mathrm{H}, 4.31 ; \mathrm{N}, 2.72 \%\right) ; \delta_{\mathrm{H}}\left(500 \mathrm{MHz} ; \mathrm{CDCl}_{3} ; \mathrm{Me}_{4} \mathrm{Si}\right) 7.90-7.78$ (4H, m, NAPH), 7.55-7.31 (8H, m, Ph, NAPH $), 7.38\left(\mathrm{~d}, 1 \mathrm{H},{ }^{3} J_{\mathrm{NH}-\mathrm{Hb}} 10 \mathrm{~Hz}, \mathrm{NH}\right) ; 7.10\left(\mathrm{~d}, 1 \mathrm{H},{ }^{3} J_{\mathrm{N} 1 \mathrm{H}-\mathrm{H} 1 \mathrm{~b}}\right.$ $\left.8.6 \mathrm{~Hz}, \mathrm{NH}_{1}\right), 5.75\left(1 \mathrm{H}, \mathrm{s}, \boldsymbol{H}_{\boldsymbol{l c}}\right), 5.25\left(1 \mathrm{H}, \mathrm{s}, \boldsymbol{H}_{\boldsymbol{c}}\right), 5.15\left(2 \mathrm{H}, \mathrm{m}, \boldsymbol{H}_{\boldsymbol{a}}, \boldsymbol{H}_{\boldsymbol{d}}\right), 5.04\left(1 \mathrm{H}, \mathrm{d},{ }^{3} J_{\mathrm{H} 1 \mathrm{a}-\mathrm{H} 1 \mathrm{~b}}\right.$ $\left.4.3 \mathrm{~Hz}, \boldsymbol{H}_{\boldsymbol{l a}}\right), 4.97\left(1 \mathrm{H}, \mathrm{d},{ }^{2} J_{\mathrm{H}-\mathrm{H}} 12 \mathrm{~Hz}, \mathrm{OCH}_{2} \mathrm{NAP}\right), 4.74\left(1 \mathrm{H}, \mathrm{d},{ }^{2} \boldsymbol{J}_{\mathrm{H}-\mathrm{H}} 12 \mathrm{~Hz}, \mathrm{OCH}_{2} \mathrm{NAP}\right), 4.62(1 \mathrm{H}, \mathrm{d}$, $\left.{ }^{2} J_{\mathrm{H}-\mathrm{H}} 11.9 \mathrm{~Hz}, \mathrm{OCH}_{2} \mathrm{Ph}\right), 4.55\left(1 \mathrm{H}, \mathrm{d},{ }^{2} J_{\mathrm{H}-\mathrm{H}} 11.9 \mathrm{~Hz}, \mathrm{OCH}_{2} \mathrm{Ph}\right), 4.30-4.18\left(4 \mathrm{H}, \mathrm{m}, \boldsymbol{H}_{\boldsymbol{b}}, \boldsymbol{H}_{\boldsymbol{l}}, \boldsymbol{H}_{\boldsymbol{l}}, \boldsymbol{H}_{\boldsymbol{g}}\right)$, $4.10\left(1 \mathrm{H}, \mathrm{d}, \boldsymbol{H}_{\boldsymbol{l d}}\right), 3.95\left(1 \mathrm{H}, \mathrm{dd}, \boldsymbol{H}_{\boldsymbol{l f}}\right), 3.80\left(2 \mathrm{H}, \mathrm{m}, \boldsymbol{H}_{\boldsymbol{f}}, \boldsymbol{H}_{\boldsymbol{l g}}\right), 3.63\left(1 \mathrm{H}, \mathrm{d},{ }^{3} \boldsymbol{J}_{\mathrm{He}-\mathrm{Hf}} 11.1 \mathrm{~Hz}, \boldsymbol{H}_{\boldsymbol{e}}\right), 2.18(3 \mathrm{H}$, s, Me $), 2.15(3 \mathrm{H}, \mathrm{s}, \boldsymbol{M e}), 1.98(3 \mathrm{H}, \mathrm{s}, \boldsymbol{M e}), 1.97(3 \mathrm{H}, \mathrm{s}, \boldsymbol{M e}) ; \delta_{\mathrm{c}}\left(125 \mathrm{MHz} ; \mathrm{CDCl}_{3} ; \mathrm{Me}_{4} \mathrm{Si}\right)$ 170.22, $170.00,169.24,169.06,161.50,161.15,137.53,134.13,133.01,128.47,128.41,127.91,127.65$, 126.56, 126.44, 126.23, 125.26, 95.09, 94.91, 92.41, 73.91, 70.05, 68.46, 67.85, 67.10, 66.82, 65.84, $64.88,64.59,61.25,49.79,20.67,20.56,20.51,20.39 ; m / z\left(\mathrm{ES}^{+}\right) 1050\left(100 \%, \mathrm{M}+[\mathrm{Na}]^{+}\right)$. 


\section{Compound 17}

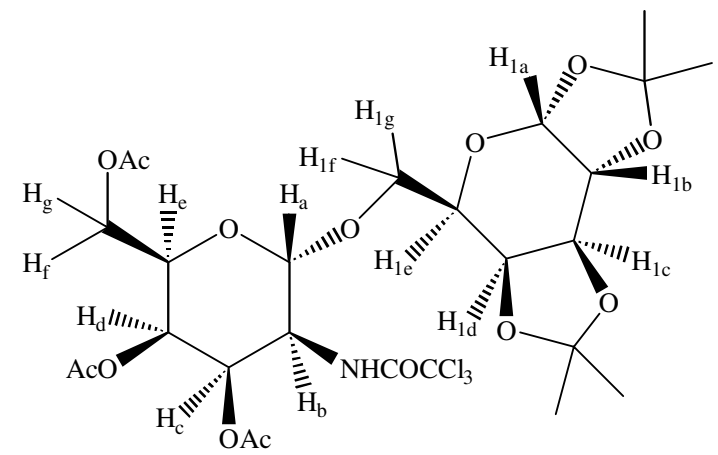

Talosamine trichloro-oxazoline glycosyl donor $14(0.265 \mathrm{~g}, 0.613 \mathrm{mmol})$ and C6-OH galactopyranose glycosyl acceptor $9(0.191 \mathrm{~g}, 0.735 \mathrm{mmol})$ were coupled using glycosidation procedure already described. The product was purified by column chromatography eluting with iso-hexane/ethyl acetate (4:1) yielding the title compound 17 as a white solid $(0.369 \mathrm{~g}, 87 \%)$. Mp. $104{ }^{\circ} \mathrm{C} ; v_{\max }(\mathrm{film}) / \mathrm{cm}^{-1}$ $3342,2987,2937,1750,1720,1529,1371,1231,1065,1046,821 ;[\alpha]_{\mathrm{D}} 19\left(\mathrm{c}=0.01, \mathrm{CHCl}_{3} @ 22^{\circ} \mathrm{C}\right)$; (Found C, 45.00; H, 5.41; N, 2.00\%, calculated for $\mathrm{C}_{26} \mathrm{H}_{36} \mathrm{NO}_{14} \mathrm{Cl}_{3}$ requires C, 45.07; H, 5.24; $\mathrm{N}, 2.02 \%) ; \delta_{\mathrm{H}}\left(500 \mathrm{MHz} ; \mathrm{CDCl}_{3} ; \mathrm{Me}_{4} \mathrm{Si}\right) 7.60\left(1 \mathrm{H}, \mathrm{d},{ }^{3} J_{\mathrm{NH}-\mathrm{Hb}} 9.6 \mathrm{~Hz}, \boldsymbol{N H}\right), 5.55\left(1 \mathrm{H}, \mathrm{d},{ }^{3} J_{\mathrm{H} 1 \mathrm{a}-\mathrm{H} 1 \mathrm{~b}} 4.95\right.$ $\left.\mathrm{Hz}, \boldsymbol{H}_{\boldsymbol{l}}\right), 5.50\left(1 \mathrm{H}, \mathrm{m}, \boldsymbol{H}_{\boldsymbol{d}}\right), 5.43\left(1 \mathrm{H}, \mathrm{t},{ }^{3} \boldsymbol{J}_{\mathrm{Hc}-\mathrm{Hd}} 4.5 \mathrm{~Hz},{ }^{3} J_{\mathrm{Hc}-\mathrm{Hb}} 4.5 \mathrm{~Hz}, \boldsymbol{H}_{\boldsymbol{c}}\right), 4.98\left(1 \mathrm{H}, \mathrm{s}, \boldsymbol{H}_{\boldsymbol{a}}\right), 4.68(1 \mathrm{H}$, $\left.\mathrm{dd},{ }^{3} J_{\mathrm{H} 1 \mathrm{c}-\mathrm{H} 1 \mathrm{~d}} 2.5 \mathrm{~Hz},{ }^{3} J_{\mathrm{H} 1 \mathrm{c}-\mathrm{H} 1 \mathrm{~b}} 7.9 \mathrm{~Hz}, \boldsymbol{H}_{\boldsymbol{l c}}\right), 4.45\left(2 \mathrm{H}, \mathrm{m}, \boldsymbol{H}_{\boldsymbol{b}}, \boldsymbol{H}_{\boldsymbol{e}}\right), 4.35\left(1 \mathrm{H}, \mathrm{dd},{ }^{3} J_{\mathrm{H} 1 \mathrm{~b}-\mathrm{H} 1 \mathrm{a}} 2.5 \mathrm{~Hz},{ }^{3} J_{\mathrm{H} 1 \mathrm{~b}-\mathrm{H} 1 \mathrm{c}}\right.$ $\left.4.95 \mathrm{~Hz}, \boldsymbol{H}_{\boldsymbol{l c}}\right), 4.27\left(2 \mathrm{H}, \mathrm{m}, \boldsymbol{H}_{\boldsymbol{l d}}, \boldsymbol{H}_{\boldsymbol{f}}\right), 4.14\left(1 \mathrm{H}, \mathrm{m}, \boldsymbol{H}_{\boldsymbol{g}}, 4.02\left(1 \mathrm{H}, \mathrm{m}, \boldsymbol{H}_{\boldsymbol{l e}}\right), 3.81\left(2 \mathrm{H}, \mathrm{m}, \boldsymbol{H}_{1 \boldsymbol{l}}, \boldsymbol{H}_{\boldsymbol{l g}}\right), 2.22\right.$ $(3 \mathrm{H}, \mathrm{s}, \boldsymbol{M e}), 2.10(3 \mathrm{H}, \mathrm{s}, \boldsymbol{M e}), 2.02$ (3H, s, Me), 1.61 (3H, s, Me), 1.45 (3H, s, Me), $1.35(6 \mathrm{H}, \mathrm{s}$, $2 \mathrm{xMe}) ; \delta_{\mathrm{c}}\left(125 \mathrm{MHz} ; \mathrm{CDCl}_{3} ; \mathrm{Me}_{4} \mathrm{Si}\right)$ 170.4, 169.23, 169.14, 161.82, 109.44, 108.75, 99.02, 96.24, 70.88, 70.64, 70.55, 67.51, 67.25, 66.64, 66.51, 64.76, 61.62, 50.48, 26.12, 25.94, 24.93, 24.36, 20.78, 20.69, 20.47; m/z (CI) $709\left(30 \%, \mathrm{M}+\left[\mathrm{NH}_{4}\right]^{+}\right)$; HRMS calculated for $\mathrm{C}_{26} \mathrm{H}_{36} \mathrm{NO}_{14} \mathrm{Cl}_{3}+\left[\mathrm{NH}_{4}\right]^{+}$requires 709.1545, found 709.1538 . 


\section{Compound 20}

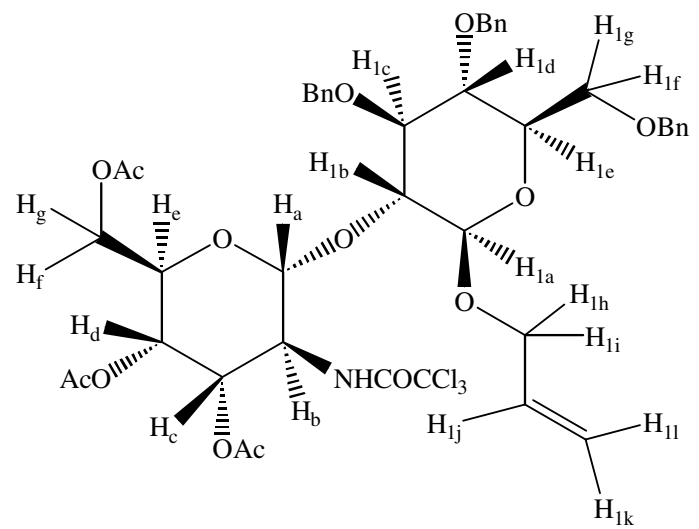

Altrosamine trichloro-oxazoline glycosyl donor $19(0.170 \mathrm{~g}, 0.3929 \mathrm{mmol})$ and C2-OH glucose glycosyl acceptor $6(0.231 \mathrm{~g}, 0.4715 \mathrm{mmol})$ were coupled using the glycosidation procedure already described. The product was purified by column chromatography eluting with iso-hexane/ethyl acetate (6:1) yielding the title compound 20 as a resin $(0.316 \mathrm{~g}, 87 \%) .[\alpha]_{\mathrm{D}} 27\left(\mathrm{c}=0.1, \mathrm{CHCl}_{3} @ 25^{\circ} \mathrm{C}\right) ; \mathrm{v}_{\max }$ (film) $/ \mathrm{cm}^{-1}$ 3330, 3029, 2931, 2866, 1748, 1721, 1525, 1366, 1231, 1060, 821, 738; (Found C, 57.24; $\mathrm{H}, 5.23 ; \mathrm{N}, 1.44 \%$, calculated for $\mathrm{C}_{44} \mathrm{H}_{50} \mathrm{NO}_{14} \mathrm{Cl}_{3}$ requires $\left.\mathrm{C}, 57.24 ; \mathrm{H}, 5.46 ; \mathrm{N}, 1.52 \%\right)$; $\delta_{\mathrm{H}}\left(400 \mathrm{MHz} ; \mathrm{CDCl}_{3} ; \mathrm{Me}_{4} \mathrm{Si}\right)$ 7.30-7.17 (13H, m, Ph), $7.02(2 \mathrm{H}, \mathrm{m}, \boldsymbol{P h}), 6.75\left(1 \mathrm{H}, \mathrm{d},{ }^{3} \boldsymbol{J}_{\mathrm{NH}-\mathrm{Hb}} 8.8 \mathrm{~Hz}\right.$, $\boldsymbol{N H}), 5.90\left(1 \mathrm{H}, \mathrm{m}, \boldsymbol{H}_{j}\right), 5.42\left(1 \mathrm{H}, \mathrm{d},{ }^{3} \boldsymbol{J}_{\mathrm{Ha}-\mathrm{Hb}} 2.4 \mathrm{~Hz}, \boldsymbol{H}_{\boldsymbol{a}}\right), 5.30\left(1 \mathrm{H}, \mathrm{t},{ }^{3} \boldsymbol{J}_{\mathrm{Hc}-\mathrm{Hd}} 9.6 \mathrm{~Hz},{ }^{3} J_{\mathrm{Hc}-\mathrm{Hb}} 9.6 \mathrm{~Hz}, \boldsymbol{H}_{\boldsymbol{c}}\right)$, $5.23\left(1 \mathrm{H}, \mathrm{m}, \boldsymbol{H}_{1 l}\right), 5.30\left(1 \mathrm{H}, \mathrm{d},{ }^{3} J_{\mathrm{H} 1 \mathrm{k}-\mathrm{H} 1 \mathrm{j}} 8.4 \mathrm{~Hz}, \boldsymbol{H}_{\boldsymbol{l}}\right), 5.08\left(1 \mathrm{H}, \mathrm{dd},{ }^{3} J_{\mathrm{Hd}-\mathrm{Hc}} 2.5 \mathrm{~Hz},{ }^{3} J_{\mathrm{Hd}-\mathrm{He}} 7.8 \mathrm{~Hz}, \boldsymbol{H}_{\boldsymbol{d}}\right)$, $5.00\left(1 \mathrm{H}, \mathrm{d},{ }^{2} J_{\mathrm{H}-\mathrm{H}} 10 \mathrm{~Hz}, \mathrm{OCH} \boldsymbol{H}_{2} \mathrm{Ph}\right), 4.90\left(1 \mathrm{H}, \mathrm{d},{ }^{2} J_{\mathrm{H}-\mathrm{H}} 10 \mathrm{~Hz}, \mathrm{OCH} \boldsymbol{H}_{2} \mathrm{Ph}\right), 4.80\left(1 \mathrm{H}, \mathrm{d},{ }^{2} J_{\mathrm{H}-\mathrm{H}} 10 \mathrm{~Hz}\right.$, $\left.\mathrm{OCH}_{2} \mathrm{Ph}\right), 4.65-4.52\left(3 \mathrm{H}, \mathrm{m}, 3 \mathrm{xOCH}_{2} \mathrm{Ph}\right), 4.42\left(1 \mathrm{H}, \mathrm{d},{ }^{3} J_{\mathrm{H} 1 \mathrm{a}-\mathrm{H} 1 \mathrm{~b}} 7.97 \mathrm{~Hz}, \boldsymbol{H}_{1 a}\right), 4.40-4.30\left(4 \mathrm{H}, \mathrm{m}, \boldsymbol{H}_{f}\right.$, $\left.\boldsymbol{H}_{\boldsymbol{g}}, \boldsymbol{H}_{\boldsymbol{b}}, \boldsymbol{H}_{\boldsymbol{l i}}\right), 4.05\left(1 \mathrm{H}, \mathrm{dd},{ }^{3} \boldsymbol{J}_{\mathrm{H} 1 \mathrm{~h}-\mathrm{H} 1 \mathrm{j}} 7.8 \mathrm{~Hz},{ }^{2} \boldsymbol{J}_{\mathrm{H} 1 \mathrm{~h}-\mathrm{H} 1 \mathrm{i}} 7.8 \mathrm{~Hz}, \boldsymbol{H}_{\boldsymbol{l h}}\right), 3.82\left(1 \mathrm{H}, \mathrm{m}, \boldsymbol{H}_{\boldsymbol{l f}}\right), 3.79-3.60(5 \mathrm{H}, \mathrm{m}$, $\left.\boldsymbol{H}_{1 b}, \boldsymbol{H}_{1 \boldsymbol{l}}, \boldsymbol{H}_{1 d}, \boldsymbol{H}_{1 g}, \boldsymbol{H}_{\boldsymbol{e}}\right), 3.40\left(1 \mathrm{H}, \mathrm{m}, \boldsymbol{H}_{1 e}\right), 2.11$ (3H, s, Me), 2.01 (3H, s, Me), 1.99 (3H, s, Me); $\delta_{\mathrm{c}}\left(75 \mathrm{MHz} ; \mathrm{CDCl}_{3} ; \mathrm{Me}_{4} \mathrm{Si}\right) 170.50,169.57,169.37,161.98,138.24,137.91,133.74,128.81,128.77$, $128.68,128.24,128.13,128.00,127.80,118.53,102.46,98.03,83.53,78.95,76.19,75.81,75.23$, $73.83,70.77,68.67,67.56,66.45,64.92,61.60,50.39,21.05,20.95,20.82 ; \mathrm{m} / z\left(\mathrm{ES}^{+}\right) 945(100 \%$, $\left.\mathrm{M}+[\mathrm{Na}]^{+}\right)$. 


\section{Compound 22}

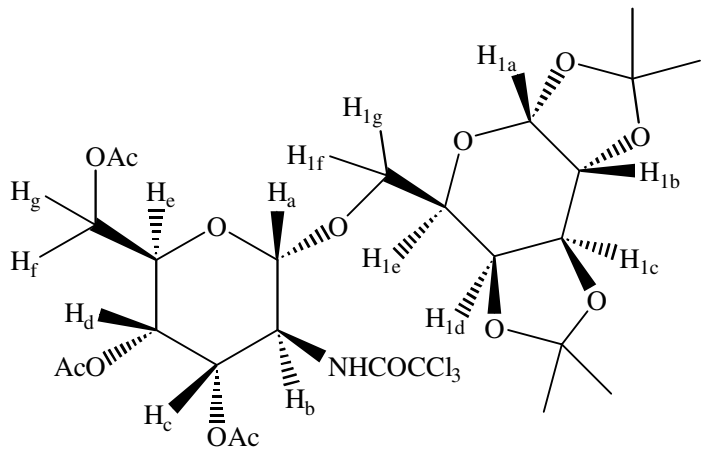

Trichloro-oxazoline glycosyl donor $19(0.392 \mathrm{~g}, 0.906 \mathrm{mmol})$ and C6-OH galactopyranose glycosyl acceptor 9 (0.283 g, $1.087 \mathrm{mmol})$ were coupled using the glycosidation procedure already described. The product was purified by column chromatography eluting with iso-hexane/ethyl acetate (4:1) yielding the title compound 22 as a white solid $(0.571 \mathrm{~g}, 91 \%) . \mathrm{Mp} 129{ }^{\circ} \mathrm{C} ;[\alpha]_{\mathrm{D}} 19\left(\mathrm{c}=0.01, \mathrm{CHCl}_{3} @\right.$ $\left.22^{\circ} \mathrm{C}\right) ; v_{\max }($ film $) / \mathrm{cm}^{-1} 3338,2989,2938,1751,1723,1530,1374,1233,1173,1069,1042,918$, 901, 822; $\delta_{\mathrm{H}}\left(400 \mathrm{MHz} ; \mathrm{CDCl}_{3} ; \mathrm{Me}_{4} \mathrm{Si}\right) 6.88\left(1 \mathrm{H}, \mathrm{d},{ }^{3} J_{\mathrm{NH}-\mathrm{Hb}} 8.7 \mathrm{~Hz}, \boldsymbol{N H}\right), 5.53\left(1 \mathrm{H}, \mathrm{d},{ }^{3} J_{\mathrm{H} 1 \mathrm{a}-\mathrm{H} 1 \mathrm{~b}} 5.1 \mathrm{~Hz}\right.$, $\left.\boldsymbol{H}_{\boldsymbol{1} \boldsymbol{a}}\right), 5.31\left(1 \mathrm{H}, \mathrm{dd},{ }^{3} J_{\mathrm{Hc}-\mathrm{Hd}} 3.5 \mathrm{~Hz},{ }^{3} J_{\mathrm{Hc}-\mathrm{Hb}} 6.0 \mathrm{~Hz}, \boldsymbol{H}_{\boldsymbol{c}}\right), 5.10\left(1 \mathrm{H}, \mathrm{dd},{ }^{3} J_{\mathrm{Hd}-\mathrm{Hc}} 3.5 \mathrm{~Hz},{ }^{3} J_{\mathrm{Hd}-\mathrm{He}} 7.8 \mathrm{~Hz}, \boldsymbol{H}_{\boldsymbol{d}}\right)$, $4.83\left(1 \mathrm{H}, \mathrm{d},{ }^{3} J_{\mathrm{Ha}-\mathrm{Hb}} 2.5 \mathrm{~Hz}, \boldsymbol{H}_{\boldsymbol{a}}\right), 4.61\left(1 \mathrm{H}, \mathrm{dd},{ }^{3} J_{\mathrm{H} 1 \mathrm{c}-\mathrm{Hid}} 2.5 \mathrm{~Hz},{ }^{3} J_{\mathrm{H} 1 \mathrm{c}-\mathrm{Hib}} 7.8 \mathrm{~Hz}, \boldsymbol{H}_{\boldsymbol{l c}}\right), 4.41\left(1 \mathrm{H}, \mathrm{m}, \boldsymbol{H}_{\boldsymbol{e}}\right)$, 4.39-4.28 (3H, m, $\left.\boldsymbol{H}_{f}, \boldsymbol{H}_{\boldsymbol{b}}, \boldsymbol{H}_{\boldsymbol{l} \boldsymbol{b}}\right), 4.30\left(2 \mathrm{H}, \mathrm{m}, \boldsymbol{H}_{\boldsymbol{g}}, \boldsymbol{H}_{\boldsymbol{l d}}\right), 4.00\left(1 \mathrm{H}, \mathrm{m}, \boldsymbol{H}_{\boldsymbol{l e}}\right), 3.72\left(1 \mathrm{H}, \mathrm{dd}, \boldsymbol{H}_{1 \boldsymbol{f}}\right), 3.69(1 \mathrm{H}$, $\left.\mathrm{dd},{ }^{3} J_{\mathrm{H} 1 \mathrm{~g}-\mathrm{H} 1 \mathrm{e}} 5.2 \mathrm{~Hz},{ }^{2} J_{\mathrm{H} 1 \mathrm{~g}-\mathrm{H} 1 \mathrm{f}} 10.0 \mathrm{~Hz}, \boldsymbol{H}_{\boldsymbol{l g}}\right), 2.20(6 \mathrm{H}, \mathrm{s}, 2 \mathrm{x} \boldsymbol{M e}), 2.08$ (3H, s, Me), 1.51 (3H, s, Me), $1.45(3 \mathrm{H}, \mathrm{s}, \boldsymbol{M e}), 1.32(6 \mathrm{H}, \mathrm{s}, 2 \mathrm{M} \boldsymbol{M}) ; \delta_{\mathrm{c}}\left(75 \mathrm{MHz} ; \mathrm{CDCl}_{3} ; \mathrm{Me}_{4} \mathrm{Si}\right)$ 170.49, 170.1, 169.65, 161.36, 109.32, 108.46, 98.92, 96.26, 70.98, 70.57, 70.33, 67.43, 66.88, 66.52, 65.43, 62.45, 52.15, 26.00, $25.91,24.83,24.47,20.64,20.56 ; \mathrm{m} / z \quad(\mathrm{CI}) 709\left(80 \%, \mathrm{M}+\left[\mathrm{NH}_{4}\right]^{+}\right) ; \quad \mathrm{HRMS}$ calculated for $\mathrm{C}_{26} \mathrm{H}_{36} \mathrm{NO}_{14} \mathrm{Cl}_{3}+\left[\mathrm{NH}_{4}\right]^{+}$requires 709.1545, found 709.1551 .

\section{Propagation of Trichloro-oxazolines}

\section{Compound 26}

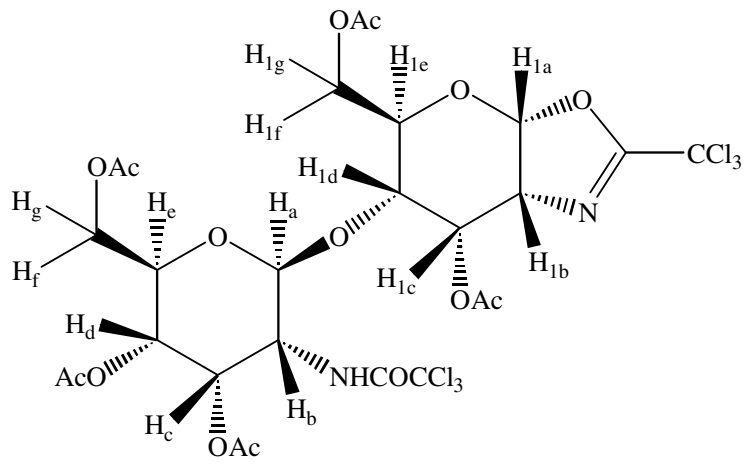

The disaccharide lactols $25(0.45 \mathrm{~g}, 0.53 \mathrm{mmol})$ were activated to the trichloro-oxazoline disaccharide glycosyl donor $\mathbf{2 6}$ using the optimised activating procedure described earlier. Purification via column 
chromatography eluting with iso-hexane/ethyl acetate $\left(2: 3,1 \% \mathrm{Et}_{3} \mathrm{~N}\right)$ affording the title compound 26 as a white amorphous solid $(0.392 \mathrm{~g}, 89 \%)$. Mp $155^{\circ} \mathrm{C} ; v_{\max }($ film $) / \mathrm{cm}^{-1} 3327,2956,1752,1525$, 1372, 1227, 1043; $[\alpha]_{\mathrm{D}}+38\left(\mathrm{c}=1, \mathrm{CHCl}_{3} @ 21^{\circ} \mathrm{C}\right) ; \delta_{\mathrm{H}}\left(500 \mathrm{MHz} ; \mathrm{CDCl}_{3} ; \mathrm{Me}_{4} \mathrm{Si}\right) 7.38\left(1 \mathrm{H}, \mathrm{d},{ }^{3} J_{\mathrm{NH}-\mathrm{Hb}}\right.$ $7.9 \mathrm{~Hz}, \mathrm{NH}), 6.21\left(1 \mathrm{H}, \mathrm{d},{ }^{3} J_{\mathrm{H} 1 \mathrm{a}-\mathrm{H} 1 \mathrm{~b}} 7.3 . \mathrm{Hz}, \boldsymbol{H}_{\boldsymbol{1 a}}\right), 5.64\left(1 \mathrm{H}, \mathrm{dd},{ }^{3} J_{\mathrm{Hc}-\mathrm{Hb}} 3.2 \mathrm{~Hz},{ }^{3} J_{\mathrm{Hc}-\mathrm{Hd}} 3.2 \mathrm{~Hz}, \boldsymbol{H}_{\boldsymbol{c}}\right), 5.50$ $\left(1 \mathrm{H}, \mathrm{dd},{ }^{3} J_{\mathrm{H} 1 \mathrm{c}-\mathrm{H} 1 \mathrm{~b}} 4.5 \mathrm{~Hz},{ }^{3} J_{\mathrm{H} 1 \mathrm{c}-\mathrm{H} 1 \mathrm{~d}} 4.5 \mathrm{~Hz}, \boldsymbol{H}_{\boldsymbol{l c}}\right), 5.04\left(1 \mathrm{H}, \mathrm{dd},{ }^{3} J_{\mathrm{Hd}-\mathrm{Hc}} 3.2 \mathrm{~Hz},{ }^{3} J_{\mathrm{Hd}-\mathrm{He}} 9.0 \mathrm{~Hz}, \boldsymbol{H}_{\boldsymbol{d}}\right), 4.80$ $\left(1 \mathrm{H}, \mathrm{d},{ }^{3} J_{\mathrm{Ha}-\mathrm{Hb}} 7.5 \mathrm{~Hz}, \boldsymbol{H}_{\boldsymbol{a}}\right), 4.64\left(1 \mathrm{H}, \mathrm{dd},{ }^{3} J_{\mathrm{Hf}-\mathrm{He}} 3.9 \mathrm{~Hz},{ }^{3} J_{\mathrm{Hf}-\mathrm{Hg}} 12.4 \mathrm{~Hz}, \boldsymbol{H}_{\boldsymbol{f}}\right), 4.37\left(1 \mathrm{H}, \mathrm{dd},{ }^{3} J_{\mathrm{H} 1 \mathrm{~b}-\mathrm{H} 1 \mathrm{c}} 4.9\right.$ $\left.\mathrm{Hz},{ }^{3} J_{\mathrm{Hlb}-\mathrm{H} 1 \mathrm{a}} 7.3 \mathrm{~Hz}, \boldsymbol{H}_{\boldsymbol{l b}}\right), 4.32-4.10\left(5 \mathrm{H}, \mathrm{m}, \boldsymbol{H}_{\boldsymbol{l} \boldsymbol{e}}, \boldsymbol{H}_{l f}, \boldsymbol{H}_{\boldsymbol{l g}}, \boldsymbol{H}_{\boldsymbol{b}}, \boldsymbol{H}_{\boldsymbol{g}}\right), 4.01\left(1 \mathrm{H}, \mathrm{ddd},{ }^{3} \boldsymbol{J}_{\mathrm{He}-\mathrm{Hf}} 3.9 \mathrm{~Hz},{ }^{3} J_{\mathrm{He}-\mathrm{Hg}}\right.$ $\left.9.0 \mathrm{~Hz},{ }^{3} J_{\mathrm{He}-\mathrm{Hd}} 9.0 \mathrm{~Hz}, \boldsymbol{H}_{\boldsymbol{e}}\right), 3.90\left(1 \mathrm{H}, \mathrm{dd},{ }^{3} J_{\mathrm{H} 1 \mathrm{~d}-\mathrm{H} 1 \mathrm{e}} 4.5 \mathrm{~Hz},{ }^{3} J_{\mathrm{H} 1 \mathrm{~d}-\mathrm{H} 1 \mathrm{e}} 8.3 \mathrm{~Hz}, \boldsymbol{H}_{\boldsymbol{l d}}\right), 2.18(3 \mathrm{H}, \mathrm{s}, \boldsymbol{M e}), 2.15$ (3H, s, Me), $2.14(3 \mathrm{H}, \mathrm{s}, \boldsymbol{M e}), 2.11(3 \mathrm{H}, \mathrm{s}, \boldsymbol{M e}), 2.00(3 \mathrm{H}, \mathrm{s}, \boldsymbol{M e}) ; \delta_{\mathrm{c}}\left(75 \mathrm{MHz} ; \mathrm{CDCl}_{3} ; \mathrm{Me}_{4} \mathrm{Si}\right)$ 171.1, 170.7, 169.5, 169.1, 168.9, 163.1, 161.4, 104.9, 100.1, 92.1, 86.2, 72.5, 71.1, 70.6, 67.3, 66.3, 65.7, 63.1, 62.8, 62.0, 53.0, 21.0, 20.8, 20.7, 20.5, 20.4; m/z (EI) 820 (10\%, M+1), 382 (25\%), 122 (100\%); HRMS calculated for $\mathrm{C}_{26} \mathrm{H}_{30} \mathrm{~N}_{2} \mathrm{O}_{15} \mathrm{Cl}_{6}$ requires 820.9855, found 820.9876 .

\section{Compound 27}

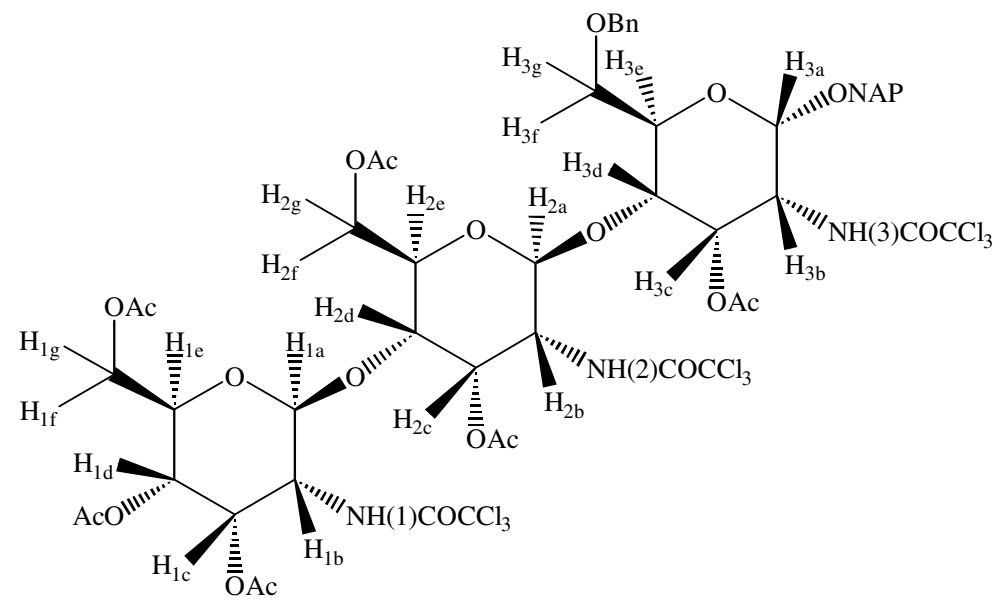

Disaccharide trichloro-oxazoline glycosyl donor $26(0.160 \mathrm{~g}, 0.194 \mathrm{mmol})$ and C4-OH glycosyl acceptor 7 (0.128 $\mathrm{g}, 0.213 \mathrm{mmol})$ were coupled using the glycosidation procedure already described. The product purified by column chromatography eluting with iso-hexane/ethyl acetate (3:2) yielding the title compound 27 as a white solid $(0.231 \mathrm{~g}, 84 \%)$. Mp $153 \mathrm{C} ; \mathrm{v}_{\max }(\mathrm{film}) / \mathrm{cm}^{-1}$ 3424, 3321, 2935, $1751,1723,1516,1371,1227,1155,1054,822 ;[\alpha]_{\mathrm{D}}+24 \quad\left(\mathrm{c}=0.011, \mathrm{CHCl}_{3} \quad\right.$ @ $\left.22{ }^{\circ} \mathrm{C}\right)$; (Found C, 45.47; H, 4.22; N, 3.09\%, calculated for $\mathrm{C}_{54} \mathrm{H}_{58} \mathrm{~N}_{3} \mathrm{O}_{22} \mathrm{Cl}_{9}$ requires $\mathrm{C}, 45.67 ; \mathrm{H}, 4.12 ; \mathrm{N}$, $2.96 \%) ; \delta_{\mathrm{H}}\left(500 \mathrm{MHz} ; \mathrm{CDCl}_{3} ; \mathrm{Me}_{4} \mathrm{Si}\right)$ 7.90-7.75 (4H, m, NAPH), $7.70\left(1 \mathrm{H}, \mathrm{d},{ }^{3} J\right.$ 7.9 Hz), 7.53-7.26 $(8 \mathrm{H}, \mathrm{m}, \boldsymbol{P h}, \boldsymbol{N A P H}), 7.16\left(1 \mathrm{H}, \mathrm{d},{ }^{3} J 8.6 \mathrm{~Hz}\right), 6.78\left(1 \mathrm{H}, \mathrm{d},{ }^{3} J 8.1 \mathrm{~Hz}\right), 5.78\left(1 \mathrm{H}, \mathrm{t},{ }^{3} J 3.2 \mathrm{~Hz},{ }^{3} J 3.2 \mathrm{~Hz}\right)$, $5.74\left(1 \mathrm{H}, \mathrm{t},{ }^{3} J 3.0 \mathrm{~Hz}\right), 5.63\left(1 \mathrm{H}, \mathrm{t},{ }^{3} J 2.9 \mathrm{~Hz}\right), 5.04\left(1 \mathrm{H}, \mathrm{d},{ }^{3} J 4.5 \mathrm{~Hz}\right), 4.95\left(1 \mathrm{H}, \mathrm{d},{ }^{2} J 11.8 \mathrm{~Hz}\right), 4.88$ (1H, dd, $\left.{ }^{3} J 3 \mathrm{~Hz}, 9.8 \mathrm{~Hz}\right), 4.82-4.67$ (5H, m), 4.52 (1H, d, $\left.{ }^{3} J 11.8 \mathrm{~Hz}\right), 4.27(1 \mathrm{H}, \mathrm{m}), 4.23-4.16(2 \mathrm{H}, \mathrm{m})$, 
4.07-3.99 (5H, m), $3.96\left(1 \mathrm{H}, \mathrm{ddd},{ }^{3} J 3 \mathrm{~Hz},{ }^{2} J 8.1 \mathrm{~Hz},{ }^{3} J 8.1 \mathrm{~Hz}\right), 3.85\left(1 \mathrm{H}, \mathrm{dt},{ }^{3} J 3 \mathrm{~Hz}, 9.8 \mathrm{~Hz}\right)$, $3.80\left(1 \mathrm{H}, \mathrm{dd},{ }^{3} J 3 \mathrm{~Hz},{ }^{2} J 11 \mathrm{~Hz}\right), 3.60\left(1 \mathrm{H}, \mathrm{d},{ }^{2} J 11 \mathrm{~Hz}\right), 3.52\left(1 \mathrm{H}, \mathrm{dd},{ }^{3} J 3 \mathrm{~Hz}, 9.8 \mathrm{~Hz}\right), 2.19(3 \mathrm{H}, \mathrm{s}$, $\boldsymbol{M e}), 2.18(3 \mathrm{H}, \mathrm{s}, \boldsymbol{M e}), 2.16(3 \mathrm{H}, \mathrm{s}, \boldsymbol{M e}), 2.10$ (3H, s, Me), 2.03 (3H, s, Me), 1.98 (3H, s, Me); $\delta_{\mathrm{c}}\left(100 \mathrm{MHz} ; \mathrm{CDCl}_{3} ; \mathrm{Me}_{4} \mathrm{Si}\right) 173.22,172.26,171.66,170.54,170.31,170.23,162.86,162.65,162.44$, $138.81,135.32,134.13,134.01,129.52,129.43,129.01,128.74,128.71,128.55,127.48,127.46$, 127.23, 126.27, 100.47, 100.03, 95.71, 92.95, 92.91, 77.37, 74.78, 74.41, 73.85, 72.07, 71.06, 70.67, 70.58, 69.63, 69.29, 68.95, 67.01, 66.94, 63.24, 62.28, 54.05, 53.95, 50.96, 21.61, 21.34, 21.13, 21.0, 20.85, 20.82; $\mathrm{m} / z\left(\mathrm{APCI}^{-}\right) 1454\left(100 \%, \mathrm{M}+[\mathrm{Cl}]^{-}\right), 1415(90 \%, \mathrm{M})$.

\section{Dehalogenation}

a) General tin hydride procedure

The trichloroacetamide $(0.0444 \mathrm{mmol})$ was added to a solution of tributyltinhydride $(0.117 \mathrm{~g}, 0.4037$ mmol) and AIBN (5 mol\%) dissolved in degassed benzene $(10 \mathrm{ml})$. The resulting contents were heated at reflux for 2 hours under an atmosphere of nitrogen, until complete by TLC analysis. Benzene was removed under reduced pressure and the crude products re-dissolved in acetonitrile $(25 \mathrm{ml})$ and washed successively with hexane $(5 \times 20 \mathrm{ml})$. The acetonitrile component was concentrated under reduced pressure to afford the crude reaction products which were purified by column chromatography eluting with methanol/ethyl acetate (1:50) yielding the title compound as a clear resin.

\section{Compound 28}

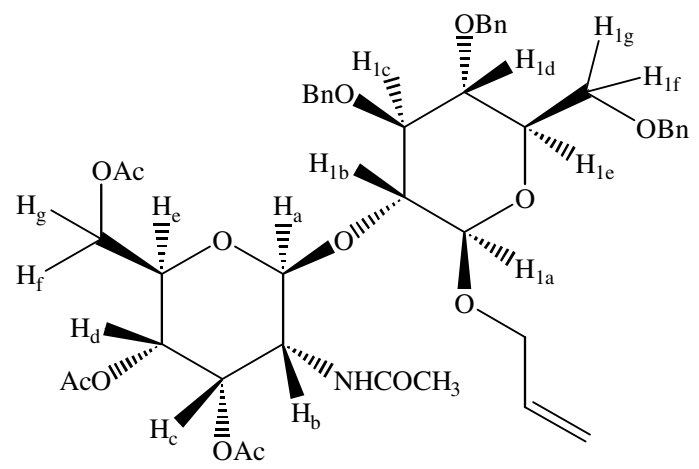

Trichloroacetamide disaccharide $11(0.045 \mathrm{~g}, 0.0487 \mathrm{mmol})$ was dechlorinated using the tin hydride procedure already described to yield the crude reaction products which were purified by column chromatography eluting with iso-hexane/ethyl acetate (1:4) yielding the title compound $\mathbf{2 8}$ as a resin $(0.037 \mathrm{~g}, 93 \%) . v_{\max }($ film $) / \mathrm{cm}^{-1} 3318,3081,3030,2906,2872,1749,1658,1547,1497,1370,1226$, 
1096, 1050, 934, 788, 736; $\delta_{\mathrm{H}}\left(400 \mathrm{MHz} ; \mathrm{CDCl}_{3} ; \mathrm{Me}_{4} \mathrm{Si}\right)$ 7.36-7.20 (15H, m, Ph), $5.90\left(1 \mathrm{H}, \mathrm{m}, \boldsymbol{H}_{\boldsymbol{k}}\right)$, $5.54\left(1 \mathrm{H}, \mathrm{t}, J 3.5 \mathrm{~Hz}, \boldsymbol{H}_{\boldsymbol{c}}\right), 5.35\left(1 \mathrm{H}, \mathrm{m}, \boldsymbol{H}_{1 l}\right), 5.25\left(1 \mathrm{H}, \mathrm{d},{ }^{3} J_{\mathrm{NH}-\mathrm{Hb}} 8.2 \mathrm{~Hz}, \mathbf{N H}\right) 5.20\left(1 \mathrm{H}, \mathrm{d},{ }^{3} J_{\mathrm{Hm}-\mathrm{Hl}} 9.6\right.$ $\left.\mathrm{Hz}, \boldsymbol{H}_{\boldsymbol{m}}\right), 5.02-4.95(3 \mathrm{H}, \mathrm{m}), 4.78\left(1 \mathrm{H}, \mathrm{d},{ }^{2} J_{\mathrm{H}-\mathrm{H}} 10 \mathrm{~Hz}, \mathrm{OCH}_{2} \mathrm{Ph}\right), 4.75\left(1 \mathrm{H}, \mathrm{d},{ }^{2} J_{\mathrm{H}-\mathrm{H}} 10 \mathrm{~Hz}, \mathrm{OC} \boldsymbol{H}_{2} \mathrm{Ph}\right)$, $4.62\left(1 \mathrm{H}, \mathrm{d},{ }^{2} \mathrm{~J}_{\mathrm{H}-\mathrm{H}} 10 \mathrm{~Hz}, \mathrm{OCH}_{2} \mathrm{Ph}\right), 4.60-4.42(4 \mathrm{H}, \mathrm{m}), 4.29-4.15$ (4H, m), 3.99 (1H, m), 3.80-3.62 (5H, m), 3.49 (1H, m), 2.02 (3H, s, Me), 2.00 (3H, s, Me), 1.95 (3H, s, Me), 1.52 (3H, s, Me); $\delta_{\mathrm{c}}\left(75 \mathrm{MHz} ; \mathrm{CDCl}_{3} ; \mathrm{Me}_{4} \mathrm{Si}\right) 171.10,169.86,169.73,169.35,138.87,138.29,138.00,134.43,128.80$, $128.68,128.65,128.18,128.12$, 128.07, 128.00, 127.81, 126.73, 116.68, 101.39, 99.99, 84.46, 81.79, 78.33, 75.08, 74.94, 73.78, 70.52, 70.30, 69.94, 68.87, 66.73, 62.83, 51.68, 23.11, 21.09, 21.01, 20.82; $m / z(\mathrm{APCI}) 842\left(100 \%, \mathrm{M}+[\mathrm{Na}]^{+}\right), 854\left(100 \%, \mathrm{M}+[\mathrm{Cl}]^{-}\right)$.

\section{b) Hydrogenolysis}

\section{Compound 29}

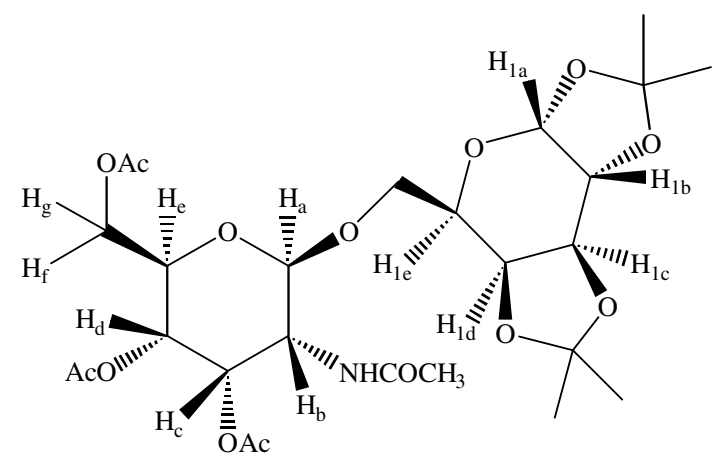

Trichloroacetamide disaccharide $12(0.065 \mathrm{~g}, 0.0866 \mathrm{mmol})$, palladium on charcoal (10\%, $10 \mathrm{~mol} \%)$ and acetic acid (1 drop) dissolved in methanol/ethanol (1:1, $20 \mathrm{ml})$ placed in a Parr hydrogenation vessel. The contents were subjected to an atmosphere of hydrogen (55 psi) for 48 hours. Triethylamine $(5 \mathrm{ml})$ was added and the palladium was removed by filtration through celite. Most of the solvent removed under reduced pressure and the crude products dissolved in ethyl acetate $(50 \mathrm{ml})$ and washed with saturated $\mathrm{NaHCO}_{3}(50 \mathrm{ml})$, water $(50 \mathrm{ml})$, dried $\mathrm{MgSO}_{4}$, and the product purified by column chromatography eluting with iso-hexane/ethyl acetate (1:9) yielding the title compound 29 as a clear gum $(0.042 \mathrm{~g}, 91 \%)$. $v_{\max }($ film $) / \mathrm{cm}^{-1} 3383,2990,2935,1748,1661,1551,1453,1374,1222,1170$, 1069, 1046, 1006, 900, 778, 731; $[\alpha]_{\mathrm{D}}-56\left(\mathrm{c}=0.01, \mathrm{CHCl}_{3} @ 22{ }^{\circ} \mathrm{C}\right) ; \delta_{\mathrm{H}}\left(400 \mathrm{MHz} ; \mathrm{CDCl}_{3} ; \mathrm{Me}_{4} \mathrm{Si}\right)$ 5.85-5.76 (2H, m, NH, $\left.\boldsymbol{H}_{\boldsymbol{c}}\right), 5.58\left(1 \mathrm{H}, \mathrm{d},{ }^{3} J_{\mathrm{H} 1 \mathrm{a}-\mathrm{H} 1 \mathrm{~b}} 5.1 \mathrm{~Hz}, \boldsymbol{H}_{\boldsymbol{1 a}}\right), 5.05\left(1 \mathrm{H}, \mathrm{dd},{ }^{3} J_{\mathrm{Hd}-\mathrm{Hc}} 2.9 \mathrm{~Hz},{ }^{3} J_{\mathrm{Hd}-\mathrm{He}} 10.1\right.$ $\left.\mathrm{Hz}, \boldsymbol{H}_{\boldsymbol{d}}\right), 4.86\left(1 \mathrm{H}, \mathrm{d},{ }^{3} J_{\mathrm{Ha}-\mathrm{Hb}} 8.6 \mathrm{~Hz}, \boldsymbol{H}_{\boldsymbol{a}}\right), 4.61\left(1 \mathrm{H}, \mathrm{dd},{ }^{3} J_{\mathrm{H} 1 \mathrm{c}-\mathrm{Hid}} 2.4 \mathrm{~Hz},{ }^{3} J_{\mathrm{H} 1 \mathrm{c}-\mathrm{Hib}} 8.04 \mathrm{~Hz}, \boldsymbol{H}_{\boldsymbol{l c}}\right), 4.25-$ $3.99\left(8 \mathrm{H}, \mathrm{m}, \boldsymbol{H}_{\boldsymbol{b}}, \boldsymbol{H}_{\boldsymbol{l b}}, \boldsymbol{H}_{\boldsymbol{e}}, \boldsymbol{H}_{\boldsymbol{f}}, \boldsymbol{H}_{\boldsymbol{g}}, \boldsymbol{H}_{\boldsymbol{l d}}, \boldsymbol{H}_{\boldsymbol{l}}, \boldsymbol{H}_{\boldsymbol{l g}}\right), 3.75\left(1 \mathrm{H}, \mathrm{dd},{ }^{3} J_{\mathrm{H} 1 \mathrm{f}-\mathrm{H} 1 \mathrm{e}} 8.2 \mathrm{~Hz},{ }^{2} J_{\mathrm{H} 1 \mathrm{f}-\mathrm{H} 1 \mathrm{~g}} 11.6 \mathrm{~Hz}, \boldsymbol{H}_{l f}\right)$, $2.20(3 \mathrm{H}, \mathrm{s}, \boldsymbol{M e}), 2.12(3 \mathrm{H}, \mathrm{s}, \boldsymbol{M e}), 2.02(6 \mathrm{H}, \mathrm{s}, 2 \times \boldsymbol{M e}), 1.55$ (3H, s, Me), 1.50 (3H, s, Me), 1.39 (6H, s, $2 \mathrm{xMe}) ; \delta_{\mathrm{c}}\left(75 \mathrm{MHz} ; \mathrm{CDCl}_{3} ; \mathrm{Me}_{4} \mathrm{Si}\right) 171.16,170.49,169.87,169.34,109.72,109.20,100.94,96.57$, 
71.15, 70.84, 70.67, 70.60, 69.90, 69.23, 68.98, 66.66, 62.72, 52.13, 26.40, 26.20, 25.30, 24.35, 23.27, $21.09\left(2 \mathrm{xC}^{13}\right), 20.84 ; \mathrm{m} / \mathrm{z}(\mathrm{APCI}) 589(100 \%, \mathrm{M}), 612\left(80 \%, \mathrm{M}+[\mathrm{Na}]^{+}\right), 623\left(100 \%, \mathrm{M}+[\mathrm{Cl}]^{-}\right)$.

c) General Two Step Dehalogenation Procedure

Trichloroacetamide disaccharide $12(0.034 \mathrm{~g}, 0.049 \mathrm{mmol})$ dissolved in ethanol $(10 \mathrm{ml})$ and $\mathrm{NaOH}(6$ eq.) was added and the reaction stirred for 10 minutes. The mixture was evaporated to dryness and pyridine added $(10 \mathrm{ml})$, acetic anhydride $(5 \mathrm{ml})$ and DMAP $(5 \mathrm{~mol} \%)$ and the contents stirred for 48 hours. The reaction was quenched with water $(20 \mathrm{ml})$, extracted with ethyl acetate $(3 \times 50 \mathrm{ml})$ dried $\left(\mathrm{MgSO}_{4}\right)$ and purified by column chromatography eluting with iso-hexane/ethyl acetate (1:9) yielding the title compound 29 as a resin $(0.023 \mathrm{~g}, 79 \%)$. All data was consistent with that already quoted for the product 29.

\section{General Scheme for the Synthesis of Compound 7}

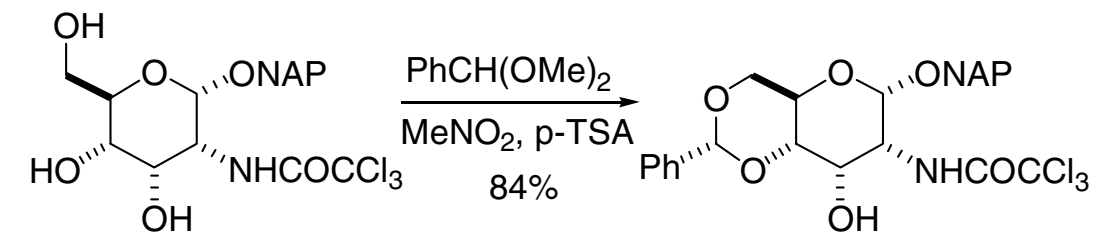

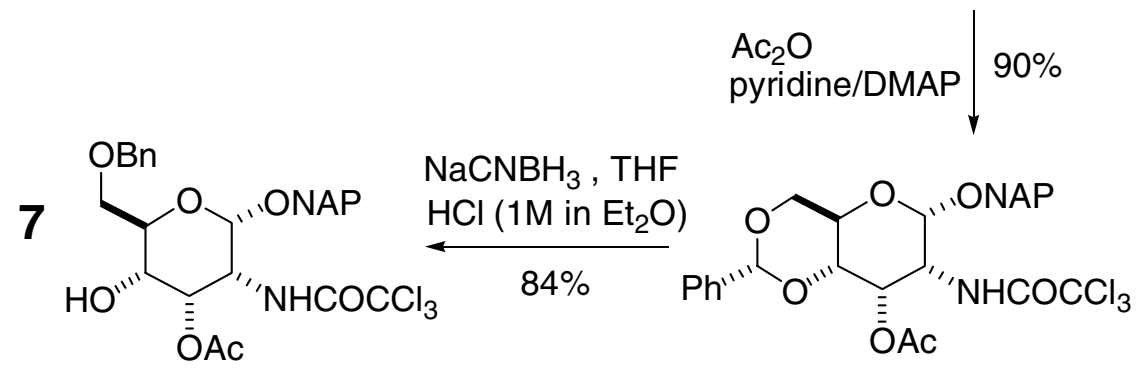


General Scheme for the Synthesis of Compounds 5 and 13

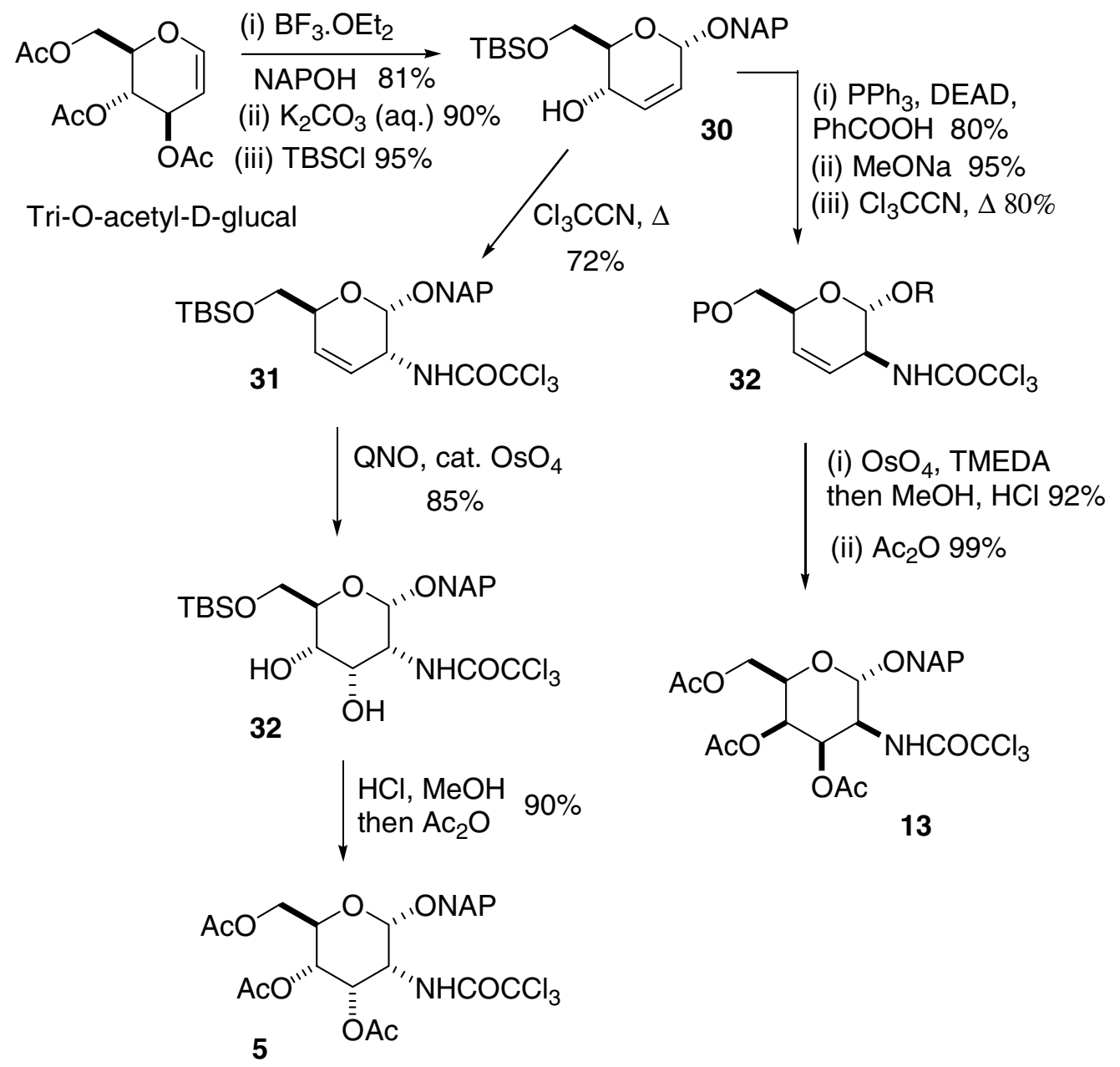

\section{Procedures for compounds 5 and 13}<smiles>CC(=O)O[C@H](C)[C@@H]1CC=C[C@@H](ON)O1</smiles>

A solution of tri- $O$-acetal- $D$-glucal $(24.72 \mathrm{~g}, 90.80 \mathrm{mmol})$ and 2-naphthalenemethanol $(15.0 \mathrm{~g}, 95.4 \mathrm{mmol})$ in DCM (450 ml) was cooled to $-28^{\circ} \mathrm{C}$ under an atmosphere of nitrogen with vigorous stirring. Boron-triflouridediethyletherate $(1.29 \mathrm{~g}, 9.09 \mathrm{mmol})$ was added and the mixture stirred for one hour at $-30{ }^{\circ} \mathrm{C}$. The contents were then allowed to warm to room temperature and stirred for a further 6 hours. The reaction could not be monitored by TLC as the reagents and products all have the same $R_{f}$ value. Reaction end point was reached when the coloured reaction mixture had become opaque, at this point the reaction was quenched with saturated $\mathrm{NaHCO}_{3}(100 \mathrm{ml})$. The products were extracted with ethyl acetate $(3 \times 100 \mathrm{ml})$, the combined organic extracts were dried $\left(\mathrm{MgSO}_{4}\right)$ removal of the solvents under reduced pressure afforded the crude reaction products as a 
brown viscous oil. The products were obtained as an anomeric mixture in a ratio of 9:1 ( $\alpha: \beta)$ as determined by NMR. The crude oil was purified by flash chromatography eluting with iso-hexane/ ethyl acetate (10:1) yielding the mixture of anomeric products as a clear oil $(31.61 \mathrm{~g}, 94 \%)$. The title compound was obtained by careful recrystallisation from cold diethyl ether triturating with iso-hexane yielded the pure title compound as fine white needles (27.24 g, 81\%) (3 crops). Mp $72{ }^{\circ} \mathrm{C} ; v_{\max }$ (film)/ cm $\mathrm{cm}^{-1} 3054(\mathrm{CH}), 2899(\mathrm{CH}), 1742(\mathrm{C}=\mathrm{O}), 1371,1237$, 1040; $[\alpha]_{D}+12\left(\mathrm{c}=1, \mathrm{CHCl}_{3} @ 23^{\circ} \mathrm{C}\right)$; (Found C, 67.77; $\mathrm{H}, 6.16 \%$, calculated for $\mathrm{C}_{21} \mathrm{H}_{22} \mathrm{O}_{6}$ requires $\mathrm{C}$, 68.10; $\mathrm{H}, 5.99 \%) ; \delta_{\mathrm{H}}\left(400 \mathrm{MHz} ; \mathrm{CDCl}_{3} ; \mathrm{Me}_{4} \mathrm{Si}\right) 7.90-7.81(4 \mathrm{H}, \mathrm{m}, \mathbf{N A P H}), 7.52-7.47(3 \mathrm{H}, \mathrm{m}, \mathbf{N A P H}), 5.93\left(1 \mathrm{H}, \mathrm{dd},{ }^{3} \mathrm{~J}_{\mathrm{Hc}-\mathrm{Hd}}\right.$ $\left.1.2 \mathrm{~Hz},{ }^{3} J_{\mathrm{Hc}-\mathrm{Hb}} 10.6 \mathrm{~Hz}, \boldsymbol{H}_{c}\right), 5.88\left(1 \mathrm{H}, \mathrm{dd},{ }^{3} J_{\mathrm{Hb}-\mathrm{Ha}} 1.6 \mathrm{~Hz},{ }^{3} J_{\mathrm{Hb}-\mathrm{Hc}} 10.6 \mathrm{~Hz}, \boldsymbol{H}_{b}\right), 5.36\left(1 \mathrm{H}, \mathrm{dd},{ }^{3} J_{\mathrm{Hd}-\mathrm{Hc}} 1.2 \mathrm{~Hz},{ }^{3} J_{\mathrm{Hd}-\mathrm{He}} 8.0\right.$ $\left.\mathrm{Hz}, \boldsymbol{H}_{\mathrm{d}}\right), 5.19\left(1 \mathrm{H}, \mathrm{s}, \boldsymbol{H}_{\mathrm{a}}\right), 4.99\left(1 \mathrm{H}, \mathrm{d},{ }^{2} \boldsymbol{J}_{\mathrm{H}-\mathrm{H}} 11.8 \mathrm{~Hz}, \mathrm{OCH}_{2} \mathrm{NAP}\right), 4.78\left(1 \mathrm{H}, \mathrm{d},{ }^{2} \boldsymbol{J}_{\mathrm{H}-\mathrm{H}} 11.8 \mathrm{~Hz}, \mathrm{OCH}{ }_{2} \mathrm{NAP}\right), 4.32-4.16$ $\left(3 \mathrm{H}, \mathrm{m}, \boldsymbol{H}_{e}, \boldsymbol{H}_{f}, \boldsymbol{H}_{g}\right), 2.11(3 \mathrm{H}, \mathrm{s}, \boldsymbol{M e}), 2.09(3 \mathrm{H}, \mathrm{s}, \boldsymbol{M e}) ; \delta_{\mathrm{c}}\left(75 \mathrm{MHz} ; \mathrm{CDCl}_{3} ; \mathrm{Me}_{4} \mathrm{Si}\right)$ 170.73, 170.22, 134.86, 133.17, 132.95, 129.30, 128.22, 127.80, 127.69, 127.62, 126.81, 126.14, 125.97, 125.83, 93.50, 70.26, 67.09, 65.26, 62.91, 20.90, 20.74; $\mathrm{m} / \mathrm{z}(\mathrm{EI}) 388\left(40 \%, \mathrm{M}^{+}\right)$; HRMS calculated for $\mathrm{C}_{21} \mathrm{H}_{22} \mathrm{O}_{6}$ requires 370.1416 , found 370.1415 .

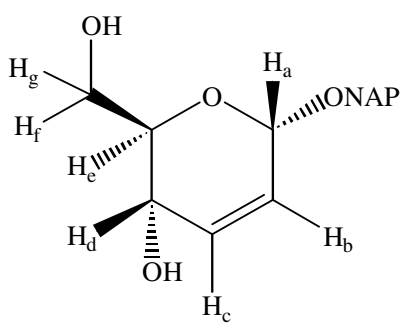

The di-acetate $(28.00 \mathrm{~g}, 77.68 \mathrm{mmol})$ dissolved in methanol/water $(4: 1),(400 \mathrm{ml})$, to this was added potassium carbonate $(1.07 \mathrm{~g}, 7.75 \mathrm{mmol})$ was added and the reaction mixture was left to stir for 16 hours at room temperature (complete by TLC analysis). Water $(100 \mathrm{ml})$ was added to the reaction with subsequent removal of the methanol under reduced pressure, the remaining aqueous component was then extracted with ethyl acetate $(3 \times 150 \mathrm{ml})$. The organic extracts were combined and washed with water $(3 \times 100 \mathrm{ml})$, dried $\left(\mathrm{MgSO}_{4}\right)$ and concentrated under reduced pressure to yield the crude product as a viscous brown oil. The crude oil was purified by flash chromatography eluting with iso-hexane/ethyl acetate (4:1) yielding the title compound as a clear gum $(20.0 \mathrm{~g}, 97 \%)$. Careful recrystallisation from cold diethyl ether/light petroleum ether afforded the diol as fine white needles (19.48 $\mathrm{g}, 90 \%)$. Mp $91^{\circ} \mathrm{C} ; v_{\max }(\mathrm{film}) / \mathrm{cm}^{-1} 3385,3051,2909,2886,1384,1321,1288$, $1121,1110,1092,1068 ; \delta_{\mathrm{H}}\left(300 \mathrm{MHz} ; \mathrm{CDCl}_{3} ; \mathrm{Me}_{4} \mathrm{Si}\right)$ 7.95-7.85 (4H, m, NAPH), 7.58-7.48 (3H, m, NAPH), 6.03 $\left(1 \mathrm{H}, \mathrm{d},{ }^{3} J_{\mathrm{Hc}-\mathrm{Hb}} 9.50 \mathrm{~Hz}, \boldsymbol{H}_{c}\right),\left(1 \mathrm{H}, \mathrm{d},{ }^{3} J_{\mathrm{Hb}-\mathrm{Hc}} 9.50 \mathrm{~Hz}, \boldsymbol{H}_{b}\right), 5.2\left(1 \mathrm{H}, \mathrm{s}, \boldsymbol{H}_{\mathrm{a}}\right), 4.95\left(1 \mathrm{H}, \mathrm{d},{ }^{2} J_{\mathrm{H}-\mathrm{H}} 12 \mathrm{~Hz}, \mathrm{OC} \boldsymbol{H}_{2} \mathrm{NAP}\right), 4.80$ $\left(1 \mathrm{H}, \mathrm{d}, J_{\mathrm{H}-\mathrm{H}} 12 \mathrm{~Hz}, \mathrm{OC} \boldsymbol{H}_{2} \mathrm{NAP}\right), 4.25\left(1 \mathrm{H}, \mathrm{d},{ }^{3} \mathrm{~J}_{\mathrm{Hd}-\mathrm{He}} 9.0 \mathrm{~Hz}, \boldsymbol{H}_{d}\right), 3.95-3.75\left(3 \mathrm{H}, \mathrm{m}, \boldsymbol{H}_{e}, \boldsymbol{H}_{t}, \boldsymbol{H}_{g}\right), 2.4(2 \mathrm{H}, \mathrm{br} \mathrm{s}, \mathrm{OH}) ; \boldsymbol{m} / \mathbf{z}$ (Cl) $304\left(30 \%, \mathrm{M}+\left[\mathrm{NH}_{4}\right]^{+}\right)$; HRMS calculated for $\mathrm{C}_{17} \mathrm{H}_{18} \mathrm{O}_{4}+[\mathrm{NH} 4]^{+}$requires 304.1549 , found 304.1542 . 


\section{Compound 30}

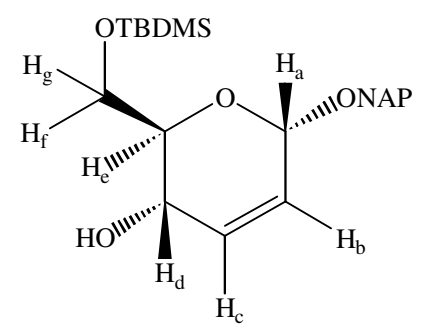

The diol (24.26 g, $84.73 \mathrm{mmol})$ was dissolved anhydrous DCM $(500 \mathrm{ml})$ and cooled to $0{ }^{\circ} \mathrm{C}$ under an atmosphere of nitrogen. To the stirred solution was added triethylamine $(18.83 \mathrm{~g}, 186.4 \mathrm{mmol})$ and the mixture stirred for 10 minutes before addition of tert-butyldimethylsilylchloride $(13.41 \mathrm{~g}, 88.97 \mathrm{~mol})$. The resulting reaction mixture was stirred for 12 hours at $0{ }^{\circ} \mathrm{C}$, warmed to room temperature and stirring continued for a further 24 hours, complete by TLC analysis. Water $(100 \mathrm{ml})$ was added prior to removal of the DCM under reduced pressure, the crude aqueous was then extracted with ethyl acetate $(3 \times 100 \mathrm{ml})$, the extracts combined and washed with saturated $\mathrm{NaHCO}_{3}(3 \times 100 \mathrm{ml})$, water $(3 \times 100 \mathrm{ml})$, dried $\left(\mathrm{MgSO}_{4}\right)$ and concentrated under reduced pressure to yield the crude product as a viscous brown oil. The resulting crude oil was further purified by column chromatography eluting with a gradient of iso-hexane/ethyl acetate $(9: 1 \rightarrow 3: 2)$ to afford the title compound 30 as a clear viscous oil (32.24 g, 95\%); $v_{\max }$ (film)/ $\mathrm{cm}^{-1} 3350 \mathrm{br}(\mathrm{OH}), 2955,2927,2864,2857,1462$, 1389, 1254; (Found C, 68.86; $\mathrm{H}, 7.97 \%$, calculated for $\mathrm{C}_{23} \mathrm{H}_{32} \mathrm{O}_{4}$ Si requires $\left.\mathrm{C}, 68.96 ; \mathrm{H}, 8.05 \%\right) ; \delta_{\mathrm{H}}(400 \mathrm{MHz}$; $\mathrm{CDCl}_{3}$; $\left.\mathrm{Me}_{4} \mathrm{Si}\right)$ 7.90-7.81 (4H, m, NAPH), 7.55-7.49 (3H, m, NAPH), $5.99\left(1 \mathrm{H}, \mathrm{dt},{ }^{4} \mathrm{~J}_{\mathrm{Hc}-\mathrm{Ha}} 1.5 \mathrm{~Hz},{ }^{3} \mathrm{~J}_{\mathrm{Hc}-\mathrm{Hd}} 1.5 \mathrm{~Hz},{ }^{3} \mathrm{~J}_{\mathrm{Hc}-\mathrm{Hb}}\right.$ $\left.10.0 \mathrm{~Hz}, \boldsymbol{H}_{c}\right), 5.80\left(1 \mathrm{H}\right.$, ddd, J $\left.2.5 \mathrm{~Hz}, \boldsymbol{J} 3.0 \mathrm{~Hz},{ }^{3} \boldsymbol{J}_{\mathrm{Hb}-\mathrm{Hc}} 10.0 \mathrm{~Hz}, \boldsymbol{H}_{b}\right), 5.12\left(1 \mathrm{H}, \mathrm{m}, \boldsymbol{H}_{\mathrm{a}}\right), 4.95\left(1 \mathrm{H}, \mathrm{d},{ }^{2} J_{\mathrm{H}-\mathrm{H}} 12.0 \mathrm{~Hz}\right.$, OCH $\left.\boldsymbol{H}_{2} \mathrm{NAP}\right), 4.79\left(1 \mathrm{H}, \mathrm{d},{ }^{2} \mathrm{~J}_{\mathrm{H}-\mathrm{H}} 12.0 \mathrm{~Hz}, \mathrm{OCH} \boldsymbol{H}_{2} \mathrm{NAP}\right), 4.22-4.20\left(1 \mathrm{H}, \mathrm{m}, \boldsymbol{H}_{d}\right), 3.85-3.75\left(3 \mathrm{H}, \mathrm{m}, \boldsymbol{H}_{e}, \boldsymbol{H}_{f} \boldsymbol{H}_{g}\right), 3.0(1 \mathrm{H}$, d, $\left.{ }^{3} \mathrm{~J}_{\text {ОН-Hd }} 5.0 \mathrm{~Hz}, \mathrm{OH}\right), 0.95\left(9 \mathrm{H}, \mathrm{s}\right.$, tert-Butyl), $0.11(3 \mathrm{H}, \mathrm{s}, \mathbf{M e}), 0.09(3 \mathrm{H}, \mathrm{s}, \mathbf{M e}) ; \delta_{\mathrm{c}}\left(100 \mathrm{MHz} ; \mathrm{CDCl}_{3} ; \mathrm{Me}_{4} \mathrm{Si}\right)$ 135.48, 133.19, 133.0, 132.89, 128.09, 127.81, 127.62, 126.65, 126.05, 125.93, 125.84, 125.74, 93.50, 70.24, $70.17,66.96,65.19,25.81,18.20,-5.55,-5.61 ; \mathrm{m} / \mathrm{z}(\mathrm{Cl}) 418\left(90 \%, \mathrm{M}+\left[\mathrm{NH}_{4}\right]^{+}\right)$; HRMS calculated for $\mathrm{C}_{23} \mathrm{H}_{32} \mathrm{O}_{4} \mathrm{Si}+\left[\mathrm{NH}_{4}^{+}\right]$requires 418.2413 , found 418.2408.

\section{Compound 31}

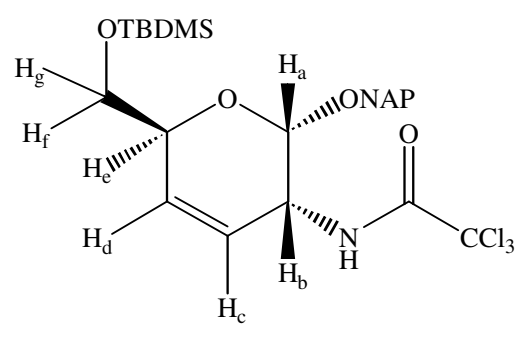


Allylic alcohol $30(28.45 \mathrm{~g}, 71.13 \mathrm{mmol})$ and anhydrous DCM $(100 \mathrm{ml})$ charged to a two necked flask equipped with a dropping funnel and the resulting mixture cooled to $-78{ }^{\circ} \mathrm{C}$ under an atmosphere of nitrogen. Cooled DBU (71.13 mmol.) was added carefully from the dropping funnel to a rapidly stirred solution. On complete addition of DBU the reaction was stirred for a further 10 minutes at $-78{ }^{\circ} \mathrm{C}$ before rapid addition of trichloroacetonitrile $(67.4 \mathrm{mmol})$ via syringe. The resulting reaction mixture was stirred for 1 hour at $-78^{\circ} \mathrm{C}$ before slowly warming to room temperature and stirring for a further 3 hours (complete by TLC analysis). The reaction was subsequently quenched with saturated $\mathrm{NaHCO}_{3}(100 \mathrm{ml})$ followed by removal of the DCM under reduced pressure affording a crude aqueous sludge, which was extracted with ethyl acetate $(3 \times 100 \mathrm{ml})$. The organic extracts were combined and washed with brine $(100 \mathrm{ml})$, water $(3 \times 100 \mathrm{ml})$, dried $\left(\mathrm{MgSO}_{4}\right)$ and concentrated under reduced pressure to yield a crude reddish oil. The crude product was purified by column chromatography eluting with a gradient of light petroleum/diethyl ether $(9: 1 \rightarrow 4: 1)$ yielding the imidate as a clear gum (34.86g, 90\%). The resulting pure product was dried extensively by repeated azeotroping with anhydrous toluene such that the water content of the product was <20ppm.

Di-phenyl ether was freshly dried by storing over $\mathrm{MgSO}_{4}$ for 24 hours at $35^{\circ} \mathrm{C}$ prior to filtering and successive azeotroping with anhydrous toluene, such that the water content of the reaction solvent was $<25 \mathrm{ppm}$. A four necked flask equipped with an overhead mechanical stirrer fitted with a Teflon boat paddle, internal temperature probe, dropping funnel and reflux condenser fitted with a drying tube was charged with di-phenyl ether (320 ml, 40 vol.), (where $1 \mathrm{vol}$. is defined as 1 gram of substrate in $1 \mathrm{ml}$ of the reaction solvent). Potassium carbonate $(0.3 \mathrm{~mol} \%)$ added and the resulting mixture heated to $130{ }^{\circ} \mathrm{C}$ (internal temperature) for 2 hours with stirring under a positive atmosphere of dry nitrogen. Pre-dried imidate $(8.00 \mathrm{~g}, 14.68 \mathrm{mmol})$ dissolved in diphenyl ether $(10 \mathrm{ml})$ was charged to the dropping funnel, at the same time the contents of the reaction vessel were quickly heated to $197^{\circ} \mathrm{C}$ (internal temperature) over a period of approximately 20 minutes. The imidate was charged to the reaction vessel when the contents had reached $170{ }^{\circ} \mathrm{C}$ and heating continued rapidly until the internal reaction temperature was exactly $197^{\circ} \mathrm{C}$. The resulting reaction mixture was continued heating at this temperature for a further 3 hours with vigorous mechanical stirring under an atmosphere of nitrogen. The reaction progress was monitored by HPLC for both consumption of the starting material and product formation. Upon complete consumption of starting material the contents were allowed to cool to room temperature and the di-phenyl ether was removed from the crude reaction products by passing through a plug of silica eluting with iso-hexane. The crude products were then further eluted from the silica with iso-hexane/ethyl acetate (3:1) and concentrated under reduced pressure to yield a viscous brown oil. Purification of the crude oil by column chromatography eluting with iso-hexane/ethyl acetate (10:1) afforded the title compound 31 as a clear oil $(6.41 \mathrm{~g}, 80 \%)$. Further purification was achieved by careful recrystallisation from cold iso-hexane yielding the allylic trichloroacetamide 31 as a white crystalline solid $(5.76 \mathrm{~g}, 72 \%)$. Mp $76{ }^{\circ} \mathrm{C} ; v_{\max }(\mathrm{film}) / \mathrm{cm}^{-1} 3420,3005$, 2961, 1717, 1505, 1369, 1221, 1041; $[\alpha]_{D}+26$ (c=1, $\left.\mathrm{CHCl}_{3} @ 23^{\circ} \mathrm{C}\right)$; (Found $\mathrm{C}, 55.35 ; \mathrm{H}, 5.72 ; \mathrm{N}, 2.56 \%$, calculated for $\mathrm{C}_{25} \mathrm{H}_{32} \mathrm{NO}_{4} \mathrm{SiCl}_{3}$ requires $\left.\mathrm{C}, 55.10 ; \mathrm{H}, 5.92 ; \mathrm{N}, 2.57 \%\right) ; \delta_{\mathrm{H}}\left(400 \mathrm{MHz} ; \mathrm{CDCl}_{3} ; \mathrm{Me}_{4} \mathrm{Si}\right) 7.88-7.78(4 \mathrm{H}$, m, NAPH), 7.54-7.44 (3H, m, NAPH), $7.13\left(1 \mathrm{H}, \mathrm{d},{ }^{3} J_{\mathrm{NH}-\mathrm{Hb}} 8.9 \mathrm{~Hz}, \mathrm{NH}\right), 5.99\left(1 \mathrm{H}, \mathrm{ddd},{ }^{4} J_{\mathrm{Hc}-\mathrm{He}} 1.9 \mathrm{~Hz},{ }^{3} \mathrm{~J}_{\mathrm{Hc} \text {-Hb }} 1.9 \mathrm{~Hz}\right.$, $\left.{ }^{3} J_{\mathrm{Hc}-\mathrm{Hd}} 10.4 \mathrm{~Hz}, \boldsymbol{H}_{c}\right), 5.66\left(1 \mathrm{H}, \mathrm{ddd},{ }^{4} \mathrm{~J}_{\mathrm{Hd}-\mathrm{Hb}} 2.0 \mathrm{~Hz},{ }^{3} \boldsymbol{J}_{\mathrm{Hd}-\mathrm{He}} 3.3 \mathrm{~Hz},{ }^{3} \mathrm{~J}_{\mathrm{Hd}-\mathrm{Hc}} 10.4 \mathrm{~Hz}, \boldsymbol{H}_{d}\right), 5.17\left(1 \mathrm{H}, \mathrm{d},{ }^{3} \boldsymbol{J}_{\mathrm{Ha}-\mathrm{Hb}} 4.5 \mathrm{~Hz}, \boldsymbol{H}_{\mathrm{a}}\right)$, $5.00\left(1 \mathrm{H}, \mathrm{d},{ }^{3} \mathrm{~J}_{\mathrm{H}-\mathrm{H}} 12.0 \mathrm{~Hz}, \mathrm{OCH}_{2} \mathrm{NAP}\right), 4.80\left(1 \mathrm{H}, \mathrm{d},{ }^{3} \mathrm{~J}_{\mathrm{H}-\mathrm{H}} 12.0 \mathrm{~Hz}, \mathrm{OCH}_{2} \mathrm{NAP}\right), 4.74-4.68\left(1 \mathrm{H}, \mathrm{m}, \boldsymbol{H}_{b}\right), 4.34-4.28$ 
$\left(1 \mathrm{H}, \mathrm{m}, \boldsymbol{H}_{e}\right), 3.80\left(1 \mathrm{H}, \mathrm{dd},{ }^{3} J_{\mathrm{HH}-\mathrm{He}} 5.8 \mathrm{~Hz},{ }^{3} \mathrm{~J}_{\mathrm{HF}-\mathrm{Hg}} 10.3 \mathrm{~Hz}, \boldsymbol{H}_{\mathrm{f}}\right), 3.68\left(1 \mathrm{H}, \mathrm{dd},{ }^{3} \mathrm{~J}_{\mathrm{Hg}-\mathrm{He}} 6.0 \mathrm{~Hz},{ }^{3} J_{\mathrm{Hg}-\mathrm{Hf}} 10.3 \mathrm{~Hz}, \boldsymbol{H}_{g}\right)$, 0.95 (9H, s, tert-Butyl), 0.12 (6H, s, 2xMe); $\delta_{\mathrm{c}}\left(75 \mathrm{MHz} ; \mathrm{CDCl}_{3} ; \mathrm{Me}_{4} \mathrm{Si}\right) 161.56,134.18,133.19,133.14,129.10$, 128.54, 127.85, 127.73, 127.23, 126.39, 126.26, 125.83, 123.05, 93.86, 69.89, 69.16, 65.28, 47.28, 25.87 (3xMe), 18.33, -5.32, $-5.41 ; \mathrm{m} / \mathrm{z}(\mathrm{Cl}) 561\left(20 \%, \mathrm{M}+\left[\mathrm{NH}_{4}\right]^{+}\right), 386(40 \%), 158(100 \%)$; HRMS calculated for $\mathrm{C}_{25} \mathrm{H}_{32} \mathrm{NO}_{4} \mathrm{SiCl}_{3}+\left[\mathrm{NH}_{4}\right]^{+}$requires 561.1510 , found 561.1516 .

\section{Compound 32}

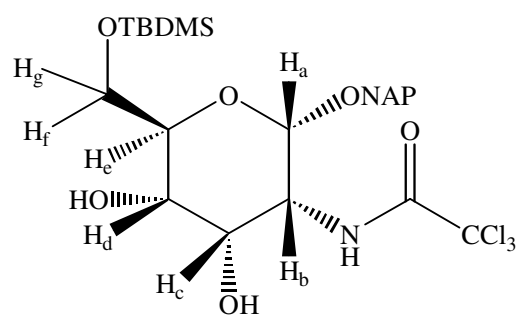

Allylic trichloroacetamide $31(0.11 \mathrm{~g}, 0.2018 \mathrm{mmol})$ and quinuclidine- $N$-oxide mono hydrate $(0.032 \mathrm{~g}, 0.22 \mathrm{mmol})$ were dissolved in anhydrous DCM $(50 \mathrm{ml})$ at room temperature under an atmosphere of nitrogen. Osmium tetraoxide $(5.0 \mathrm{~mol} \%)$ added and the resulting reaction mixture stirred for 2 days after which a black solution resulted, complete by TLC analysis. Saturated sodium sulphite $(50 \mathrm{ml})$ was added to the reaction and the contents heated to $50^{\circ} \mathrm{C}$ for 4 hours. The mixture was allowed to cool and extracted with ethyl acetate $(3 \times 100 \mathrm{ml})$ the extracts were combined, washed with saturated $\mathrm{NaHCO}_{3}(50 \mathrm{ml})$ and water $(3 \times 75 \mathrm{ml})$, dried $\left(\mathrm{MgSO}_{4}\right)$ and concentrated under reduced pressure to afford the a crude brown oil. The crude oil was purified by column chromatography eluting with iso-hexane/ethyl acetate $(20: 1)$ to afford the diol as a clear viscous oil $(0.10 \mathrm{~g}, 85 \%)$, (100\% syn isomer).

\section{Compound 5}

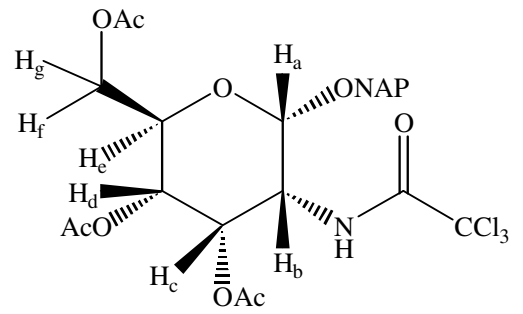

The diol $(5.65 \mathrm{~g}, 9.7585 \mathrm{mmol})$ was dissolved in methanol $(50 \mathrm{ml})$, conc. $\mathrm{HCl}$ (2 drops) was added and the resulting reaction mixture stirred for 5 hours at room temperature (complete by TLC analysis). The reaction was then quenched with saturated $\mathrm{NaHCO}_{3}(50 \mathrm{ml})$ and the methanol removed under reduced pressure, the resulting crude mixture was extracted with ethyl acetate $(3 \times 200 \mathrm{ml})$. The organics were combined washed with water $(3 \times 100 \mathrm{ml})$, dried $\left(\mathrm{MgSO}_{4}\right)$ and concentrated under reduced pressure to yield a crude coloured oil. The crude oil was purified by column chromatography eluting with iso-hexane/ethyl acetate (3:2) to afford the title compound 103 as a white amorphous solid. The triol was further purified by careful recrystallisation from cold ethyl acetate/iso-hexane yielding the triol as fine white needles $(4.13 \mathrm{~g}, 91 \%) . \mathrm{mp} 169{ }^{\circ} \mathrm{C} ; v_{\max }(\mathrm{film}) / \mathrm{cm}^{-1} 3406,1713$, 1506, 1040; $[\alpha]_{D}+110\left(\mathrm{c}=1, \mathrm{MeOH} @ 22^{\circ} \mathrm{C}\right.$ ); (Found C, 48.84; H, 4.26; N, 3.06\%, calculated for $\mathrm{C}_{19} \mathrm{H}_{20} \mathrm{NO}_{6} \mathrm{Cl}_{3}$ 
requires $\mathrm{C}, 49.11 ; \mathrm{H}, 4.34 ; \mathrm{N}, 3.01 \%) ; \delta_{\mathrm{H}}\left(400 \mathrm{MHz} ; \mathrm{CDCl}_{3} ; \mathrm{Me}_{4} \mathrm{Si}\right)$ 7.90-7.75 (4H, m, NAPH), 7.58-7.40 (3H, m, NAPH), $7.37\left(1 \mathrm{H}, \mathrm{d},{ }^{3} \mathrm{~J}_{\mathrm{NH}-\mathrm{Hb}} 8.9 \mathrm{~Hz}, \mathrm{NH}\right), 5.14\left(1 \mathrm{H}, \mathrm{d},{ }^{3} \mathrm{~J}_{\mathrm{Ha}-\mathrm{Hb}} 4.3 \mathrm{~Hz}, \boldsymbol{H}_{\mathrm{a}}\right), 4.95\left(1 \mathrm{H}, \mathrm{d},{ }^{3} J_{\mathrm{H}-\mathrm{H}} 11.8 \mathrm{~Hz}, \mathrm{OCH}_{2} \mathrm{NAP}\right), 4.73$ $\left(1 \mathrm{H}, \mathrm{d},{ }^{3} \mathrm{~J}_{\mathrm{H}-\mathrm{H}} 11.8 \mathrm{~Hz}, \mathrm{OC} \boldsymbol{H}_{2} \mathrm{NAP}\right), 4.16-4.08\left(2 \mathrm{H}, \mathrm{m}, \boldsymbol{H}_{b}, \boldsymbol{H}_{c}\right), 4.03-3.96\left(1 \mathrm{H}, \mathrm{m}, \boldsymbol{H}_{\mathrm{f}}\right), 3.95-3.88\left(1 \mathrm{H}, \mathrm{m}, \boldsymbol{H}_{g}\right), 3.81$ $\left(1 \mathrm{H}, \mathrm{ddd},{ }^{3} \mathrm{~J}_{\mathrm{Hd}-\mathrm{He}} 2.9 \mathrm{~Hz},{ }^{3} \mathrm{~J}_{\mathrm{Hd}-\mathrm{NH}} 9.6 \mathrm{~Hz},{ }^{3} J_{\mathrm{Hd}-\mathrm{Hc}} 9.6 \mathrm{~Hz}, \boldsymbol{H}_{d}\right), 3.18\left(1 \mathrm{H}, \mathrm{d},{ }^{3} \mathrm{~J}_{\mathrm{OH}-\mathrm{Hc}} 10.0 \mathrm{~Hz}, \mathrm{OH}\right), 2.60\left(1 \mathrm{H}, \mathrm{d},{ }^{3} J_{\mathrm{OH}-\mathrm{Hd}} 9.6\right.$ $\mathrm{Hz}, \mathrm{OH}), 2.0(1 \mathrm{H}$, brs, $\mathrm{OH}) ; \delta_{\mathrm{c}}\left(75 \mathrm{MHz} ; \mathrm{CDCl}_{3} ; \mathrm{Me}_{4} \mathrm{Si}\right)$ 160.7, 135.1, 128.0, 127.7, 127.6, 126.6, 126.1, 125.9, 125.8, 95.1, 89.9, 69.1, 69.0, 68.1, 66.9, 61.8, 51.6; $\mathrm{m} / \mathrm{z}(\mathrm{Cl}) 481\left(20 \%, \mathrm{M}+\left[\mathrm{NH}_{4}\right]^{+}\right), 464(10 \%, \mathrm{M}+1)$; HRMS calculated for $\mathrm{C}_{19} \mathrm{H}_{20} \mathrm{NO}_{6} \mathrm{Cl}_{3}+\left[\mathrm{NH}_{4}\right]^{+}$requires 481.0699, found 481.0695 .

The triol $(5.0 \mathrm{~g}, 10.8 \mathrm{mmol})$ dissolved in pyridine $(50 \mathrm{ml})$, to this was added acetic anhydride (10 ml), DMAP $(5 \mathrm{~mol} \%)$ and the resulting reaction mixture stirred for 2 days at room temperature under an atmosphere of nitrogen. The subsequent reaction mixture was quenched with saturated $\mathrm{NaHCO}_{3}(50 \mathrm{ml})$ and extracted with ethyl acetate $(3 \times 100 \mathrm{ml})$. The extracts were combined, dried $(\mathrm{MgSO} 4)$ and concentrated under reduced pressure yielding the crude products as dark viscous oil. The crude oil was purified by column chromatography eluting with iso-hexane/ethyl acetate (4:1) yielding the title compound 5 as a clear viscous oil $(6.25 \mathrm{~g}, 99 \%)$. $v_{\max }(\mathrm{film}) / \mathrm{cm}^{-1} 3335,1767,1713,1506,1040 ;[\alpha]_{\mathrm{D}}+37$ (c=0.01, $\mathrm{CHCl}_{3} @ 22{ }^{\circ} \mathrm{C}$ ); (Found C, 51.14; H, 4.39; N, $2.21 \%$, calculated for $\mathrm{C}_{25} \mathrm{H}_{26} \mathrm{NO}_{9} \mathrm{Cl}_{3}$ requires $\left.\mathrm{C}, 50.84 ; \mathrm{H}, 4.40 ; \mathrm{N}, 2.37 \%\right) ; \delta_{\mathrm{H}}\left(400 \mathrm{MHz} ; \mathrm{CDCl}_{3} ; \mathrm{Me}_{4} \mathrm{Si}\right) 7.88-$ $7.78(4 \mathrm{H}, \mathrm{m}, \boldsymbol{N A P H}), 7.54-7.44(3 \mathrm{H}, \mathrm{m}, \mathbf{N A P H}), 7.17\left(1 \mathrm{H}, \mathrm{d},{ }^{3} J_{\mathrm{NH}-\mathrm{Hb}} 8.9 \mathrm{~Hz}, \mathrm{NH}\right), 5.64\left(1 \mathrm{H}, \mathrm{dd},{ }^{3} J_{\mathrm{HC}-\mathrm{Hb}} 3.4 \mathrm{~Hz},{ }^{3} \mathrm{~J}_{\mathrm{Hc}-\mathrm{Hd}}\right.$ $\left.3.4 \mathrm{~Hz}, \boldsymbol{H}_{c}\right), 5.08-5.02\left(2 \mathrm{H}, \mathrm{m}, \boldsymbol{H}_{\mathrm{a}}, \boldsymbol{H}_{\mathrm{d}}\right), 5.00\left(1 \mathrm{H}, \mathrm{d},{ }^{3} J_{\mathrm{H}-\mathrm{H}} 12.1 \mathrm{~Hz}, \mathrm{OCH}_{2} \mathrm{NAP}\right), 4.75\left(1 \mathrm{H}, \mathrm{d},{ }^{3} J_{\mathrm{H}-\mathrm{H}} 12.1 \mathrm{~Hz}\right.$, OC $\left.\boldsymbol{H}_{2} \mathrm{NAP}\right), 4.40-4.28\left(3 \mathrm{H}, \mathrm{m}, \boldsymbol{H}_{f} \boldsymbol{H}_{e}, \boldsymbol{H}_{b}\right), 4.17\left(1 \mathrm{H}, \mathrm{dd},{ }^{3} \mathrm{~J}_{\mathrm{Hg}-\mathrm{He}} 1.9 \mathrm{~Hz},{ }^{3} \mathrm{~J}_{\mathrm{Hg}-\mathrm{Hf}} 12.0 \mathrm{~Hz}, \boldsymbol{H}_{g}\right), 2.16(3 \mathrm{H}, \mathrm{s}, \boldsymbol{M e}), 2.11$ $(3 \mathrm{H}, \mathrm{s}, \mathbf{M e}), 2.0(3 \mathrm{H}, \mathrm{s}, \mathbf{M e}) ; \delta_{\mathrm{c}}\left(75 \mathrm{MHz} ; \mathrm{CDCl}_{3} ; \mathrm{Me}_{4} \mathrm{Si}\right)$ 170.6, 170.0, 169.1, 161.0, 133.9, 133.1, 133.0, 128.5, 127.8, 127.7, 126.6, 126.5, 126.3, 125.2, 94.7, 92.0, 69.9, 67.2, 65.6, 63.7, 61.9, 49.3, 20.7, 20.6, 20.5; $\mathrm{m} / \mathrm{z}(\mathrm{Cl}) 611\left(20 \%, \mathrm{M}+\left[\mathrm{NH}_{4}\right]^{+}\right), 434(50 \%)$; HRMS calculated for $\mathrm{C}_{25} \mathrm{H}_{26} \mathrm{NO}_{9} \mathrm{Cl}_{3}$ requires 593.0774, found 593.0771 .

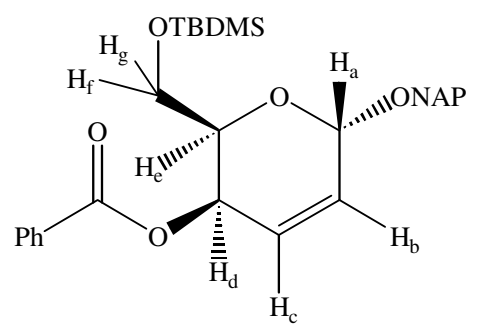

A stirred solution of the allylic alcohol $30(1.64 \mathrm{~g}, 4.09 \mathrm{mmol})$, triphenylphosphine $(4.83 \mathrm{~g}, 18.4 \mathrm{mmol})$ and benzoic acid $(1.50 \mathrm{~g}, 12.30 \mathrm{mmol})$ dissolved in anhydrous tetrahyrdrofuran $(50 \mathrm{ml})$ was cooled to $0{ }^{\circ} \mathrm{C}$ under an atmosphere of nitrogen. Di-isopropyl-diazo-dicacarboxylate $(3.56 \mathrm{~g}, 20.45 \mathrm{mmol})$ was added drop wise using a syringe pump, on complete addition the resulting reaction mixture was stirred for a further hour at $0{ }^{\circ} \mathrm{C}$. The reaction was then allowed to warm to room temperature and stirring continued for a further 4 hours, complete by TLC analysis. The reaction was quenched with saturated $\mathrm{NaHCO}_{3}(100 \mathrm{ml})$ and the crude mixture extracted with ethyl acetate $(3 \times 100 \mathrm{ml})$. The organic extracts were combined and washed successively with brine $(3 \times 100 \mathrm{ml})$ and water $(3 \times 100 \mathrm{ml})$, dried $\left(\mathrm{MgSO}_{4}\right)$ and concentrated under reduced pressure to afford the crude reaction 
products as a viscous coloured oil. The crude oil was re-dissolved in diethyl ether $(50 \mathrm{ml})$ and cooled to $0{ }^{\circ} \mathrm{C}$ which allowed for precipitation of some tri-phenylphosineoxide by-products which were filtered off, washed with cold diethyl ether and the washings concentrated under reduced pressure to yield the crude products as a red oil. The crude oil was further purified by column chromatography eluting with a gradient of iso-hexane/diethyl ether $(9: 1 \rightarrow 4: 1)$ yielding the title compound as a clear oil $(1.65 \mathrm{~g}, 80 \%) ; v_{\max }\left(\right.$ film)/ cm $\mathrm{cm}^{-1} 3056,2929,2884,2862$ $(\mathrm{CH}), 1719(\mathrm{C}=\mathrm{O}), 1602,1466,1389,1343,1270,1183,1116,1035 ;[\alpha]_{\mathrm{D}}-162\left(\mathrm{c}=0.008, \mathrm{CHCl}_{3} @ 22^{\circ} \mathrm{C}\right)$; $\delta_{\mathrm{H}}\left(400 \mathrm{MHz} ; \mathrm{CDCl}_{3} ; \mathrm{Me}_{4} \mathrm{Si}\right)$ 8.10-8.05 (2H, m, $\left.\boldsymbol{A r}\right), 7.90-7.83(4 \mathrm{H}, \mathrm{m}, \boldsymbol{A r}), 7.60-7.42(6 \mathrm{H}, \mathrm{m}, \boldsymbol{A r}), 6.33(1 \mathrm{H} . \mathrm{ddd}$, $\left.{ }^{3} \mathrm{~J}_{\mathrm{Hc}-\mathrm{Ha}} 1.0 \mathrm{~Hz},{ }^{3} \mathrm{~J}_{\mathrm{Hc}-\mathrm{Hd}} 5.5 \mathrm{~Hz},{ }^{3} \mathrm{~J}_{\mathrm{HC}-\mathrm{Hb}} 10.0 \mathrm{~Hz}, \boldsymbol{H}_{c}\right), 6.11\left(1 \mathrm{H} . \mathrm{ddd},{ }^{4} \mathrm{~J}_{\mathrm{Hb}-\mathrm{Hd}} 0.5 \mathrm{~Hz},{ }^{3} \mathrm{~J}_{\mathrm{Hb}-\mathrm{Ha}} 3.0 \mathrm{~Hz},{ }^{3} \mathrm{~J}_{\mathrm{Hb}-\mathrm{Hc}} 10.0 \mathrm{~Hz}, \boldsymbol{H}_{\mathrm{b}}\right)$, $5.32\left(1 \mathrm{H}, \mathrm{ddd},{ }^{3} \mathrm{~J}_{\mathrm{Hd}-\mathrm{Hb}} 0.5 \mathrm{~Hz},{ }^{3} J_{\mathrm{Hd}-\mathrm{He}} 2.5 \mathrm{~Hz},{ }^{3} \mathrm{~J}_{\mathrm{Hd}-\mathrm{Hc}} 15.5 \mathrm{~Hz}, \boldsymbol{H}_{d}\right), 5.28\left(1 \mathrm{H}, \mathrm{dd},{ }^{4} J_{\mathrm{Ha}-\mathrm{Hc}} 1.0 \mathrm{~Hz},{ }^{3} \boldsymbol{J}_{\mathrm{Ha}-\mathrm{Hb}} 3.0 \mathrm{~Hz}, \boldsymbol{H}_{\mathrm{a}}\right), 5.03$ $\left(1 \mathrm{H}, \mathrm{d},{ }^{2} J_{\mathrm{H}-\mathrm{H}} 12.0 \mathrm{~Hz}, \mathrm{OCH}_{2} \mathrm{NAP}\right), 4.85\left(1 \mathrm{H}, \mathrm{d},{ }^{2} J_{\mathrm{H}-\mathrm{H}} 12.0 \mathrm{~Hz}, \quad \mathrm{OCH} \mathrm{H}_{2} \mathrm{NAP}\right), 4.42\left(1 \mathrm{H} . \mathrm{dt},{ }^{3} J_{\mathrm{He}-\mathrm{Hd}} 2.5 \mathrm{~Hz},{ }^{3} J_{\mathrm{He}-\mathrm{Hig}}\right.$ $\left.7.0 \mathrm{~Hz}, \boldsymbol{H}_{\mathrm{e}}\right), 3.84\left(2 \mathrm{H}, 2 \mathrm{xdd},{ }^{3} J_{\mathrm{HF}-\mathrm{He}} 7.0 \mathrm{~Hz},{ }^{3} \mathrm{~J}_{\mathrm{Hg}-\mathrm{He}} 7.0 \mathrm{~Hz},{ }^{2} J_{\mathrm{HF}-\mathrm{Hg}} 10.0 \mathrm{~Hz},{ }^{2} J_{\mathrm{Hg}-\mathrm{Hf}} 10.0 \mathrm{~Hz} \boldsymbol{H}_{f}, \boldsymbol{H}_{g}\right), 0.85(9 \mathrm{H}, \mathrm{s}$, tertButyl), $0.02(3 \mathrm{H}, \mathrm{s}, \mathbf{M e}),-0.02(3 \mathrm{H}, \mathrm{s}, \mathrm{Me}) ; \delta_{\mathrm{c}}\left(75 \mathrm{MHz} ; \mathrm{CDCl}_{3} ; \mathrm{Me}_{4} \mathrm{Si}\right) 165.77,135.23,133.23,132.98,130.65$, 130.00, 129.64, 128.30, 128.19, 127.84, 127.65, 126.90, 126.09, 126.02, 125.89, 93.21, 69.88, 69.76, 63.30, 61.72, 18.08, -5.55, -5.65; $\mathrm{m} / \mathrm{z}\left(\mathrm{ES}^{+}\right) 527\left(100 \%, \mathrm{M}+[\mathrm{Na}]^{+}\right)$.

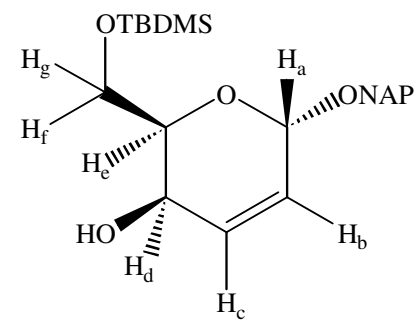

To a solution of the benzoate ester $(1.62 \mathrm{~g}, 3.21 \mathrm{mmol})$ in methanol $(50 \mathrm{ml})$ was added to freshly prepared sodium methoxide solution $(100 \mathrm{ml}, 0.1607 \mathrm{M})$ and the resulting reaction mixture stirred for 16 hours at room temperature, complete by TLC analysis. The mixture was reduced to a crude sludge under reduced pressure, water $(50 \mathrm{ml})$ was added and the crude mixture extracted with ethyl acetate $(3 \times 100 \mathrm{ml})$. The organic extracts were combined and washed with brine $(100 \mathrm{ml})$, water $(3 \times 100 \mathrm{ml})$, dried $\left(\mathrm{MgSO}_{4}\right)$ and concentrated under reduced pressure yieldeding the crude product as a viscous oil. The crude oil was purified by column chromatography eluting with a gradient of iso-hexane/diethyl ether $(9: 1 \rightarrow 3: 2)$ yielding the title compound as a colourless oil (1.22 g, 95\%). $v_{\max }$ (film)/ $\mathrm{cm}^{-1} 3449(\mathrm{OH}), 3052,2929$ and $2857(\mathrm{CH}), 1466,1390,1254,1093$, $1029 ; \delta_{\mathrm{H}}\left(300 \mathrm{MHz} ; \mathrm{CDCl}_{3} ; \mathrm{Me}_{4} \mathrm{Si}\right)$ 7.76-7.69 (4H, m, NAPH), 7.43-7.35 (3H, m, NAPH), 6.09 (1H, dd, ${ }^{3} J_{\mathrm{Hc} \cdot \mathrm{Hd}} 5.5$ $\left.\mathrm{Hz},{ }^{3} J_{\mathrm{Hc} \cdot \mathrm{Hb}} 10.0 \mathrm{~Hz}, \boldsymbol{H}_{c}\right), 5.85\left(1 \mathrm{H}, \mathrm{dd},{ }^{3} \boldsymbol{J}_{\mathrm{Hb}-\mathrm{Ha}} 3.0 \mathrm{~Hz},{ }^{3} \boldsymbol{J}_{\mathrm{Hb}-\mathrm{Hc}} 10.0 \mathrm{~Hz}, \boldsymbol{H}_{\mathrm{b}}\right), 5.09\left(1 \mathrm{H}, \mathrm{d},{ }^{3} J_{\mathrm{Ha}-\mathrm{Hb}} 3.0 \mathrm{~Hz}, \boldsymbol{H}_{\mathrm{a}}\right), 4.88(1 \mathrm{H}, \mathrm{d}$, $\left.{ }^{2} J_{H-H} 12.0 \mathrm{~Hz}, \mathrm{OCH}_{2} \mathrm{NAP}\right), 4.67\left(1 \mathrm{H}, \mathrm{d},{ }^{2} J_{H-H} 12.0 \mathrm{~Hz}, \mathrm{OC} \boldsymbol{H}_{2} \mathrm{NAP}\right), 4.08-3.99\left(1 \mathrm{H}, \mathrm{m}, \boldsymbol{H}_{d}\right), 3.87-3.67\left(3 \mathrm{H}, \mathrm{m}, \boldsymbol{H}_{e}, \boldsymbol{H}_{f}\right.$ $\left.\boldsymbol{H}_{g}\right), 2.11\left(1 \mathrm{H}, \mathrm{d},{ }^{3} \mathrm{~J}_{\mathrm{OH}-\mathrm{Hd}} 8.0 \mathrm{~Hz}, \mathrm{OH}\right), 0.82\left(9 \mathrm{H}, \mathrm{s}\right.$, tert-Butyl), $0.01(3 \mathrm{H}, \mathrm{s}, \mathbf{M e}), 0.00(3 \mathrm{H}, \mathrm{s}, \mathbf{M e}) ; \delta_{\mathrm{c}}(100 \mathrm{MHz}$; $\mathrm{CDCl}_{3}$; $\left.\mathrm{Me}_{4} \mathrm{Si}\right)$ 135.25, 133.20, 132.93, 129.77, 128.40, 128.13, 127.82, 127.62, 126.89, 126.05, 125.87, 93.36, $70.78,69.67,62.77,61.84,25.81,18.21,-5.43,-5.48 ; \mathrm{m} / \mathrm{z}(\mathrm{EI}) 418\left(20 \%, \mathrm{M}+\left[\mathrm{NH}_{4}\right]^{+}\right), 400\left(10 \%, \mathrm{M}^{+}\right)$; HRMS calculated for $\mathrm{C}_{23} \mathrm{H}_{32} \mathrm{O}_{4} \mathrm{Si}+\left[\mathrm{H}^{+}\right]$requires 418.2148 , found 418.2152 . 


\section{Compound 32}

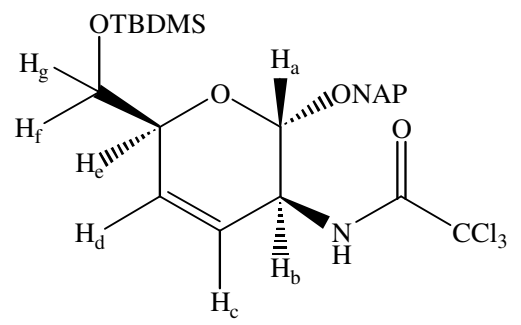

The allylic alcohol $(4.48 \mathrm{~g}, 11.12 \mathrm{mmol})$ and anhydrous DCM $(100 \mathrm{ml})$ charged to a two necked flask equipped with a dropping funnel and the resulting mixture cooled to $-78{ }^{\circ} \mathrm{C}$ under an atmosphere of nitrogen. When cooled DBU was added carefully from the dropping funnel to a rapidly stirred solution. On complete addition of DBU the reaction was stirred for a further 10 minutes at $-78{ }^{\circ} \mathrm{C}$ before rapid addition of trichloroacetonitrile $(1.53 \mathrm{~g}, 10.63 \mathrm{mmol})$ via syringe. The resulting reaction mixture was stirred for 1 hour at $-78^{\circ} \mathrm{C}$ before slowly warming to room temperature and stirring for a further 3 hours (complete by TLC analysis). The reaction was subsequently quenched with saturated $\mathrm{NaHCO}_{3}(100 \mathrm{ml})$ followed by removal of the DCM under reduced pressure affording a crude aqueous sludge, which was extracted with ethyl acetate $(3 \times 100 \mathrm{ml})$. The organic extracts were combined and washed with brine $(100 \mathrm{ml})$, water $(3 \times 100 \mathrm{ml})$, dried $\left(\mathrm{MgSO}_{4}\right)$ and concentrated under reduced pressure to yield a crude reddish oil. The crude oil was purified by column chromatography eluting with a gradient of iso-hexane/ethyl acetate $(20: 1 \rightarrow 10: 1)$ yielding the imidate as a clear oil $(5.64 \mathrm{~g}, 92 \%)$. The resulting pure product was dried extensively by repeated azeotroping with anhydrous toluene such that the water content was <20ppm. This material was unstable and used immediately in the next step of the synthesis. To a rapidly stirred solution of anhydrous xylene $(50 \mathrm{ml})$ was added potassium carbonate $(5 \mathrm{~mol} \%)$ and the trichloroacetimidate $(5.60 \mathrm{~g}, 10.28 \mathrm{mmol})$. The resulting mixture was heated to reflux under an atmosphere of nitrogen for 24 hours (complete by TLC analysis). Removal of the solvents under reduced pressure yielded the crude mixture which was re-dissolved in ethyl acetate $(100 \mathrm{ml})$ and washed with saturated $\mathrm{NaHCO}_{3}(100 \mathrm{ml})$, water $(3 \times 50 \mathrm{ml})$, dried $\mathrm{MgSO}_{4}$ and concentrated under reduced pressure to afford the crude reaction products as a brown oil. The crude oil was purified by flash chromatography eluting with light petroleum/diethyl ether (5:1), concentrated under reduced pressure, re-dissolved in diethyl ether and carefully recrystallised by triturating with light petroleum ether to afford the title compound 32 as a powdery white solid $(4.91 \mathrm{~g}, 87 \%)$, (3 crops). Mp $87^{\circ} \mathrm{C} ; v_{\max }$ (film) / cm $\mathrm{cm}^{-1} 3344,2961,1752,1525,1369,1221,1041 ;[\alpha]_{D}+110\left(\mathrm{c}=0.1, \mathrm{CHCl}_{3} @ 23^{\circ} \mathrm{C}\right) ;($ Found $\mathrm{C}, 55.34 ; \mathrm{H}, 5.64 ; \mathrm{N} 2.48 \%$, calculated for $\mathrm{C}_{25} \mathrm{H}_{32} \mathrm{NO}_{4} \mathrm{SiCl}_{3}$ requires $\left.\mathrm{C}, 55.10 ; \mathrm{H}, 5.92 ; \mathrm{N}, 2.57 \%\right) ; \delta_{\mathrm{H}}(400 \mathrm{MHz}$; $\mathrm{CDCl}_{3}$; $\left.\mathrm{Me}_{4} \mathrm{Si}\right)$ 7.90-7.80 (4H, m, NAPH), 7.51-7.46 (3H, m, NAPH), $6.67\left(1 \mathrm{H}, \mathrm{d},{ }^{3} \mathrm{~J}_{\mathrm{NH}-\mathrm{Hb}} 8.0 \mathrm{~Hz}, \mathrm{NH}\right), 6.08(1 \mathrm{H}, \mathrm{d}$, $\left.{ }^{3} J_{H d-H c} 10.0 \mathrm{~Hz}, \boldsymbol{H}_{d}\right), 5.94\left(1 \mathrm{H}, \mathrm{m}, \boldsymbol{H}_{c}\right), 5.04\left(1 \mathrm{H}, \mathrm{s}, \boldsymbol{H}_{\mathrm{a}}\right), 4.96\left(1 \mathrm{H}, \mathrm{d},{ }^{2} \boldsymbol{J}_{\mathrm{H}-\mathrm{H}} 12.0 \mathrm{~Hz}, \mathrm{OCH}_{2} \mathrm{NAP}\right), 4.82\left(1 \mathrm{H}, \mathrm{d},{ }^{2} J_{\mathrm{H}-\mathrm{H}} 12.0\right.$ $\left.\mathrm{Hz}, \mathrm{OCH}_{2} \mathrm{NAP}\right), 4.42\left(1 \mathrm{H}, \mathrm{m}, \boldsymbol{H}_{b}\right), 4.30\left(1 \mathrm{H}, \mathrm{m}, \boldsymbol{H}_{e}\right), 3.78\left(2 \mathrm{H}, 2 \mathrm{xdd},{ }^{3} J_{\mathrm{H}-\mathrm{He}} 5.0 \mathrm{~Hz},{ }^{3} J_{\mathrm{Hg}-\mathrm{He}} 5.5 \mathrm{~Hz},{ }^{2} \boldsymbol{J}_{\mathrm{H}-\mathrm{Hg}} 11.0 \mathrm{~Hz}\right.$, $\left.{ }^{2} J_{\mathrm{Hg}-\mathrm{Hf}} 11.0 \mathrm{~Hz}, \boldsymbol{H}_{f}, \boldsymbol{H}_{g}\right), 0.93\left(9 \mathrm{H}, \mathrm{s}\right.$, tert-Butyl), $0.1(3 \mathrm{H}, \mathrm{s}, \boldsymbol{M e}),-0.06(3 \mathrm{H}, \mathrm{s}, \boldsymbol{M e}) ; \delta_{\mathrm{c}}\left(100 \mathrm{MHz} ; \mathrm{CDCl}_{3} ; \mathrm{Me}_{4} \mathrm{Si}\right)$ 161.30, 134.52, 133.23, 133.19, 133.02, 131.95, 128.32, 127.91, 127.67, 126.69, 126.15, 126.00, 125.68, 121.29, 97.63, 70.27, 69.13, 64.93, 47.04, 18.43, -5.27; $\mathrm{m} / \mathrm{z}(\mathrm{Cl}) 561\left(40 \%, \mathrm{M}+\left[\mathrm{NH}_{4}\right]^{+}\right)$; HRMS calculated for $\mathrm{C}_{25} \mathrm{H}_{32} \mathrm{NO}_{4} \mathrm{SiCl}_{3}+\left[\mathrm{NH}_{4}\right]^{+}$requires 561.1510 , found 561.1512 . 


\section{Compound 13}

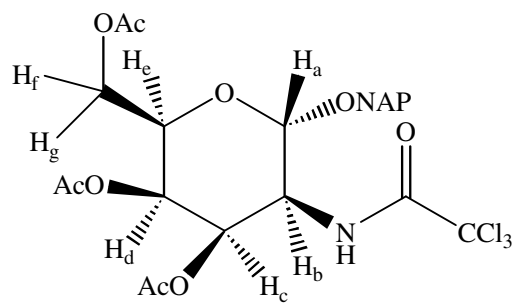

Allylic trichloroacetamide $32(1.00 \mathrm{~g}, 1.84 \mathrm{mmol})$ and TMEDA $(0.23 \mathrm{~g}, 2.02 \mathrm{mmol})$ dissolved in anhydrous DCM $(50 \mathrm{ml})$ was cooled to $-78^{\circ} \mathrm{C}$ under an atmosphere of nitrogen. A solution of $\mathrm{OsO}_{4}(0.47 \mathrm{~g}, 1.84 \mathrm{mmol})$ in DCM $(2 \mathrm{ml})$ was added immediately and the colour quickly turned deep red then brown/black. The reaction was stirred for 2 hours at $-78{ }^{\circ} \mathrm{C}$ before warming up to room temperature, complete by TLC analysis. Methanol $(50 \mathrm{ml})$ was added, and then conc. $\mathrm{HCl}(3$ drops) was added and the resulting mixture stirred for 4 hours at room temperature (complete by TLC analysis). Saturated sodium sulphite $(50 \mathrm{ml})$ was added and the resulting mixture stirred for a further hour before removal of the methanol under reduced pressure. The crude mixture was extracted with ethyl acetate $(3 \times 100 \mathrm{ml})$, the extracts were combined and washed with brine $(100 \mathrm{ml})$, water $(3 \times 100 \mathrm{ml})$, dried $\left(\mathrm{MgSO}_{4}\right)$ and concentrated under reduced pressure affording a brown solid. The crude solid was purified by column chromatography eluting with methanol/diethyl ether (1:19) yielding the triol as a white amorphous solid $(0.75 \mathrm{~g}, 92 \%)$. Mp $96^{\circ} \mathrm{C} ; v_{\max }($ film $) / \mathrm{cm}^{-1} 3406,1713,1506,1040 ;[\alpha]_{\mathrm{D}}+49\left(\mathrm{c}=0.002, \mathrm{CHCl}_{3} @\right.$ $22{ }^{\circ} \mathrm{C}$ ); (Found $\mathrm{C}, 49.13 ; \mathrm{H}, 4.44 ; \mathrm{N}, 2.98 \%$, calculated for $\mathrm{C}_{19} \mathrm{H}_{20} \mathrm{NO}_{6} \mathrm{Cl}_{3}$ requires $\left.\mathrm{C}, 49.11 ; \mathrm{H}, 4.34 ; \mathrm{N}, 3.01 \%\right) ; \delta_{\mathrm{H}}$ (300 MHz; $\mathrm{CDCl}_{3}$; $\left.\mathrm{Me}_{4} \mathrm{Si}\right) 8.50\left(1 \mathrm{H}, \mathrm{d},{ }^{3} \mathrm{~J}_{\mathrm{NH}-\mathrm{Hb}} 9.1 \mathrm{~Hz}, \mathrm{NH}\right), 7.90-7.82(4 \mathrm{H}, \mathrm{m}, \mathbf{N A P H}), 7.58-7.49(3 \mathrm{H}, \mathrm{m}, \mathbf{N A P H})$, $5.12\left(1 \mathrm{H}, \mathrm{s}, \boldsymbol{H}_{\mathrm{a}}\right), 4.86\left(1 \mathrm{H}, \mathrm{d},{ }^{2} \boldsymbol{J}_{\mathrm{H} \cdot \mathrm{H}} 11.9 \mathrm{~Hz}, \mathrm{OCH}_{2} \mathrm{NAP}\right), 4.76\left(1 \mathrm{H}, \mathrm{d},{ }^{2} J_{\mathrm{H}-\mathrm{H}} 11.9 \mathrm{~Hz}, \mathrm{OCH}_{2} \mathrm{NAP}\right), 4.40\left(1 \mathrm{H}, \mathrm{m}, \boldsymbol{H}_{b}\right)$, $4.20\left(1 \mathrm{H}, \mathrm{m}, \boldsymbol{H}_{c}\right), 4.02-3.84\left(4 \mathrm{H}, \mathrm{m}, \boldsymbol{H}_{d}, \boldsymbol{H}_{e}, \boldsymbol{H}_{f} \boldsymbol{H}_{g}\right), 2.80-2.40(3 \mathrm{H}, \mathrm{br}, 3 \times \mathrm{OH}) ; \delta_{\mathrm{c}}\left(75 \mathrm{MHz} ; \mathrm{CDCl}_{3} ; \mathrm{Me}_{4} \mathrm{Si}\right) 163.13$, 134.37, 133.44, 128.71, 128.18, 128.02, 127.11, 126.66, 126.53, 125.95, 99.32, 71.74, 70.61, 69.21, 65.08, 64.57, 53.48; $\mathrm{m} / \mathrm{z}(\mathrm{Cl}) 481\left(20 \%, \mathrm{M}+\left[\mathrm{NH}_{4}\right]^{+}\right)$; HRMS calculated for $\mathrm{C}_{19} \mathrm{H}_{20} \mathrm{NO}_{6} \mathrm{Cl}_{3}+\left[\mathrm{NH}_{4}\right]^{+}$requires 481.0699, found 481.0693.

The triol $(0.70 \mathrm{~g}, 1.5 \mathrm{mmol})$ was dissolved in pyridine $(50 \mathrm{ml})$, to this was added acetic anhydride $(10 \mathrm{ml})$ and a catalytic amount of DMAP $(5 \mathrm{~mol} \%)$ and the resulting reaction mixture was stirred for 2 days at room temperature under an atmosphere of nitrogen. The reaction was quenched with saturated $\mathrm{NaHCO}_{3}(50 \mathrm{ml})$ and extracted with ethyl acetate $(3 \times 100 \mathrm{ml})$ the organics were combined and washed with water $(3 \times 100 \mathrm{ml})$, dried (MgSO4) and concentrated under reduced pressure yielding a dark viscous oil. The crude oil was purified by column chromatography eluting with iso-hexane/ethyl acetate (4:1) to afford the title compound 13 as a clear viscous oil $(0.88 \mathrm{~g}, 99 \%) . \quad v_{\max }(\mathrm{film}) / \mathrm{cm}^{-1} 3418,2930,1754,1712,1516,1371,1227,1121,1073,1021 ;[\alpha]^{22}$ $+58\left(\mathrm{c}=0.1, \mathrm{CHCl}_{3} @ 22{ }^{\circ} \mathrm{C}\right) ; \delta_{\mathrm{H}}\left(500 \mathrm{MHz} ; \mathrm{CDCl}_{3} ; \mathrm{Me}_{4} \mathrm{Si}\right) 7.90-7.82(4 \mathrm{H}, \mathrm{m}, \mathbf{N A P H}), 7.58\left(1 \mathrm{H}, \mathrm{d},{ }^{2} J_{\mathrm{NH}-\mathrm{Hb}} 8.0 \mathrm{~Hz}\right.$, $\mathrm{NH})$, 7.53-7.49 (3H, m, NAPH), 5.47-5.42 (2H, m, $\left.\boldsymbol{H}_{c}, \boldsymbol{H}_{d}\right), 5.05\left(1 \mathrm{H}, \mathrm{brs}, \boldsymbol{H}_{\mathrm{a}}\right), 4.88\left(1 \mathrm{H}, \mathrm{d},{ }^{2} \boldsymbol{J}_{\mathrm{H}-\mathrm{H}} 9.50 \mathrm{~Hz}\right.$, $\left.\mathrm{OCH}_{2} \mathrm{NAP}\right), 4.75\left(1 \mathrm{H}, \mathrm{d},{ }^{2} J_{\mathrm{H}-\mathrm{H}} 9.50 \mathrm{~Hz}, \mathrm{OCH}_{2} \mathrm{NAP}\right), 4.60\left(1 \mathrm{H}, \mathrm{dd},{ }^{3} J_{\mathrm{Hb}-\mathrm{Hc}} 3.5 \mathrm{~Hz},{ }^{3} J_{\mathrm{Hb}-\mathrm{NH}} 8.0 \mathrm{~Hz}, \boldsymbol{H}_{\mathrm{b}}\right), 4.45(1 \mathrm{H}, \mathrm{dd}$, $\left.{ }^{3} J_{\mathrm{He}-\mathrm{HH}} 5.0 \mathrm{~Hz},{ }^{3} J_{\mathrm{He}-\mathrm{Hg}} 5.0 \mathrm{~Hz}, \boldsymbol{H}_{e}\right), 4.20\left(2 \mathrm{H}, 2 \mathrm{xdd},{ }^{3} J_{\mathrm{HT}-\mathrm{He}} 5.0 \mathrm{~Hz},{ }^{3} J_{\mathrm{Hg}-\mathrm{He}} 5.0 \mathrm{~Hz},{ }^{2} J_{\mathrm{H}-\mathrm{Hg}} 9.0 \mathrm{~Hz},{ }^{2} J_{\mathrm{Hg}-\mathrm{Hf}} 9.0 \mathrm{~Hz}, \boldsymbol{H}_{\boldsymbol{f}}, \boldsymbol{H}_{g}\right), 2.30$ $(3 \mathrm{H}, \mathrm{s}, \mathbf{M e}), 2.20(3 \mathrm{H}, \mathrm{s}, \mathbf{M e}), 2.10(3 \mathrm{H}, \mathrm{s}, \mathbf{M e}) ; \delta_{\mathrm{c}}\left(125 \mathrm{MHz} ; \mathrm{CDCl}_{3} ; \mathrm{Me}_{4} \mathrm{Si}\right)$ 170.78, 169.68, 169.53, 162.22, 
133.75, 133.61, 133.56, 129.03, 128.38, 128.18, 127.69, 126.83, 126.76, 126.22, 98.67, 70.58, 67.76, 67.18, $65.24, \quad 62.09, \quad 50.96,21.21,21.08,20.89 ; \quad \mathrm{m} / \mathrm{z}(\mathrm{Cl}) 607 \quad\left(25 \%, \mathrm{M}+\left[\mathrm{NH}_{4}\right]^{+}\right) ; \mathrm{HRMS}$ calculated for $\mathrm{C}_{25} \mathrm{H}_{26} \mathrm{NO}_{9} \mathrm{Cl}_{3}+\left[\mathrm{NH}_{4}\right]^{+}$requires 607.1016 , found 607.1019 . 